\title{
Rab33a Mediates Anterograde Vesicular Transport for Membrane Exocytosis and Axon Outgrowth
}

\author{
Hitomi Nakazawa, ${ }^{1}$ Tadayuki Sada, ${ }^{1}$ Michinori Toriyama, ${ }^{1}$ Kenji Tago, ${ }^{2}$ Tadao Sugiura, ${ }^{3}$ Mitsunori Fukuda, ${ }^{4}$ \\ and Naoyuki Inagaki ${ }^{1}$ \\ Laboratories of ${ }^{1}$ Neuronal Cell Morphogenesis and ${ }^{2}$ Molecular Signal Transduction, Graduate School of Biological Sciences, and ${ }^{3}$ Laboratory of Biomedical \\ Imaging and Informatics, Graduate School of Information Science, Nara Institute of Science and Technology, Ikoma, Nara 630-0192, Japan, and \\ ${ }^{4}$ Laboratory of Membrane Trafficking Mechanisms, Graduate School of Life Sciences, Tohoku University, Sendai, Miyagi 980-8578, Japan
}

\begin{abstract}
Axon outgrowth requires plasma membrane expansion, which results from post-Golgi vesicular transport and fusion. However, the molecular mechanisms regulating post-Golgi vesicular trafficking for membrane expansion and axon outgrowth remain unclear. Here, we show that Rab33a expression became upregulated during axon outgrowth of cultured rat hippocampal neurons. Rab33a was preferentially localized to the Golgi apparatus and to synaptophysin-positive vesicles that are transported along the growing axon. Previous studies showed that synaptophysin is localized to post-Golgi vesicles transported by fast axonal transport in developing neurons. Reduction of Rab33a expression by RNAi (RNA interference) inhibited the anterograde transport of synaptophysin-positive vesicles, leading to their decrease in axonal tips. Furthermore, this treatment reduced membrane fusion of synaptophysin-positive vesicles at the growth cones and inhibited axon outgrowth. Overexpression of Rab33a, on the other hand, induced excessive accumulation of synaptophysinpositive vesicles and concurrent formation of surplus axons. These data suggest that Rab33a participates in axon outgrowth by mediating anterograde axonal transport of synaptophysin-positive vesicles and their concomitant fusion at the growth cones.
\end{abstract}

\section{Introduction}

Throughout axon outgrowth, neurons undergo dramatic expansion of the plasma membrane. An axon of $1 \mu \mathrm{m}$ in diameter growing at a rate of $500 \mu \mathrm{m} / \mathrm{d}$ must expand its surface membrane by $1500 \mu \mathrm{m}^{2} / \mathrm{d}$, while the surface membrane area of a cell body $10 \mu \mathrm{m}$ in diameter is $\sim 400 \mu \mathrm{m}^{2}$. The rough endoplasmic reticulum and Golgi apparatus in the neuronal cell body serve as the primary site for the generation of plasmalemmal precursor vesicles (Futerman and Banker, 1996; Pfenninger, 2009; Sann et al., 2009). Although less is known about the vesicular fusion events that underlie axonal membrane expansion, a number of studies suggest that growth cones are sites of exocytosis and membrane expansion for axon outgrowth (Bray, 1970; Lockerbie et al., 1991; Craig et al., 1995; Igarashi et al., 1996; Pfenninger, 2009; Eva et al., 2010; Bloom and Morgan, 2011; Wang et al., 2011).

Received Feb. 29, 2012; revised July 11, 2012; accepted July 25, 2012.

Author contributions: H.N., T. Sada, M.T., K.T., M.F., and N.I. designed research; H.N., T. Sada, and M.T. performed research; H.N., T. Sada, and T. Sugiura analyzed data; H.N. and N.I. wrote the paper.

This research was supported in part by the Ministry of Education, Culture, Sports, Science, and Technology (MEXT) and Japan Society for the Promotion of Science Grants-in-Aid for Scientific Research (KAKENHI), the Global COE (Centers of Excellence) Program at Nara Institute of Science and Technology (NAIST) (MEXT), Osaka Medical Research Foundation for Incurable Diseases, and NAIST Interdisciplinary Frontier Research Project. We thank Drs. Yuichi Sakumura, Michihiro Igarashi, Hiroshi Itoh, Sadao Shiosaka, and Yasumasa Bessho for discussions, Dr. lan Smith for reviewing the manuscript, and Dr. Masayoshi Matsumoto for technical advice.

The authors declare no competing financial interests.

Correspondence should be addressed to Naoyuki Inagaki, Laboratory of Neuronal Cell Morphogenesis, Graduate School of Biological Sciences, Nara Institute of Science and Technology, Ikoma, Nara 630-0192, Japan. E-mail: ninagaki@bs.naist.jp.

DOI:10.1523/JNEUROSCI.0989-12.2012

Copyright $\odot 2012$ the authors $\quad 0270-6474 / 12 / 3212712-14 \$ 15.00 / 0$
Rab small GTPases have been shown to play crucial roles in the regulation of intracellular vesicular trafficking pathways (Pfeffer, 2001; Zerial and McBride, 2001; Fukuda, 2008). Rab proteins function as molecular switches by cycling between GTPbound active and GDP-bound inactive states. Particular Rab proteins localize to distinct intracellular vesicles and organelles, thereby regulating their specific trafficking for vesicular budding, transport, docking, and fusion. Recent studies have identified Rab proteins that are involved in vesicular trafficking underlying axon outgrowth. For example, Rab10 regulates the insertion of plasmalemmal precursor vesicles at the growth cones for axon outgrowth (Wang et al., 2011). Rab7, Rab27 and Rab11 participate in the axonal transport of the neurotrophin receptors Trk and $\mathrm{p} 75^{\mathrm{NTR}}$, which regulate neurite outgrowth (Deinhardt et al., 2006; Arimura et al., 2009; Ascaño et al., 2009). However, diverse vesicular compartments containing distinct subsets of proteins are transported along the axon, and the molecules regulating the post-Golgi pathways that govern membrane expansion and axon outgrowth remain unclear (Pfenninger, 2009).

The Rab family protein Rab33a is expressed in the brain, lymphocytes, and melanocytes (Zheng et al., 1997; Cheng et al., 2006; Lee et al., 2006), but its functions are largely unknown. We previously reported that Singar1/Rufy3, a RUN domain-containing protein, suppresses the formation of surplus axons in cultured rat hippocampal neurons, thereby contributing to the robustness of neuronal polarity (Mori et al., 2007). In this study, based on our recent identification of an interaction between Rab33a and Singar1 (Fukuda et al., 2011), we examined the possible involvement of Rab33a in axon outgrowth. We show that Rab33a is localized to the Golgi apparatus and to synaptophysin-positive vesicles 
that are transported along growing axons. Synaptophysin is localized to post-Golgi vesicles transported by fast axonal transport in developing neurons (Fletcher et al., 1991; Phelan and GordonWeeks, 1992; Nakata et al., 1998; Rosso et al., 2004); at the growth cones, synaptophysin-positive vesicles are thought be added to the plasma membrane for membrane expansion (Igarashi et al., 1997). Our data suggest that Rab33a mediates the anterograde axonal transport of these post-Golgi synaptophysin-positive vesicles, thereby contributing to membrane insertion at the growth cones and to axon outgrowth.

\section{Materials and Methods}

Cultures, transfection, and drug treatment. Hippocampal neurons prepared from embryonic day 18 (E18) rat embryos were seeded on coverslips coated with poly-D-lysine (Sigma-Aldrich) and laminin (Iwaki), and cultured in Neurobasal medium (Invitrogen) supplemented with B27 supplement (Invitrogen) and $1 \mathrm{~mm}$ glutamine without a glia feeder layer, as described previously (Mori et al., 2007). The cells were transfected with vectors using Nucleofector (Lonza) before plating. For nocodazole treatment, hippocampal neurons cultured for $48 \mathrm{~h}$ were incubated with $7 \mu \mathrm{M}$ nocodazole (Sigma-Aldrich) for $2 \mathrm{~h}$ before fixation.

cDNA constructs. Full-length cDNA of rat Rab33a was amplified by PCR from a rat brain cDNA library (Clontech Laboratories) with the primers 5'-GCATGTCGACATGGCGCAGCCCATCCTGGGCCATGG-3' and 5'-GCATGTCGACTCAACAAGGACAGGAAGTTTTACTGTTAGC-3'. Preparation of cDNA of mouse Rab33a Q95L (a constitutive active mutant) was reported previously (Itoh et al., 2006). cDNA of an inactive rat Rab33a mutant was produced by making a threonine-to-arginine substitution (T50R; Kobayashi et al., 2009) using the QuikChange II Site-Directed Mutagenesis Kit (Stratagene) with the primers $5^{\prime}$-TCCAACGTGGGCAAGCGC TGCCTG-3' and 5'-TCAGGCAGCGCTTGCCCACGTTGG-3'. Fulllength cDNA of rat synaptophysin was amplified with the primers $5^{\prime}$-TAGG ATCCATGGACGTGGTGAATCAGCTGG-3' and 5'-GCGGATCCTTAC ATCTGATTGGAGAAGGAG-3'. The cDNAs were then subcloned into pCAGGS-Myc with a $\beta$-actin promoter (provided by J. Miyazaki, Osaka University, Osaka, Japan; Niwa et al., 1991), pEGFP-C1 (Clontech Laboratories), and pmRFP (provided by Dr. R. Tsien, University of California, San Diego, La Jolla, CA) vectors.

Immunoaffinity purification of synaptophysin-containing vesicles. Purification of synaptophysin-containing vesicles was performed as described previously (Fukuda, 2004). Briefly, Dynabeads M-280 coated covalently with sheep anti-mouse IgG (Dynal) was incubated with antisynaptophysin antibody (H-8; Santa Cruz Biotechnology) or control mouse IgG. Hippocampal neurons were homogenized and incubated with magnetic beads coated with primary antibody. The bound fractions were analyzed by immunoblot.

RNAi. For vector-based RNAi (RNA interference) of Rab33a, we used the BLOCK-iT Pol II miR RNAi Expression Vector kit (Invitrogen). The targeting microRNA (miRNA) sequence1 5'-CAGGACAAGAACGCTT CCGTA-3' corresponds to nucleotides 278-298 in the coding region of rat Rab33a and sequence $25^{\prime}$-TGACCTCCTTCACCAACTTAA-3' corresponds to 365-385, whereas the control vector pcDNA 6.2-GW/ EmGFP-miR-neg encodes an mRNA which does not target any known vertebrate gene. To ensure high-level expression of miRNA before neurite extension, hippocampal neurons prepared from E18 rat embryos and transfected with miRNA were cultured on uncoated polystyrene plates. After a $24 \mathrm{~h}$ incubation to induce miRNA expression, the cells were collected and cultured on coverslips coated with polyD-lysine and laminin.

Immunocytochemistry, immunoblot, and microscopy. Immunocytochemistry and immunoblot were performed as described previously (Mori et al., 2007). For 5-chloromethylfluorescein diacetate (CMFDA) and 7-amino-4chloromethylcoumarin (CMAC) staining, neurons were incubated with CMFDA (1:500 dilution) and CMAC (1:2000 dilution; both from Invitrogen) for $2 \mathrm{~h}$ before fixation. Fluorescence images of neurons were acquired using an Axioplan2 fluorescence microscope (Carl Zeiss) equipped with a Plan-Apochromat $63 \times$ oil, 1.40 NA (numerical aperture), a PlanNEOFLUAR $40 \times, 0.75 \mathrm{NA}$ and $20 \times, 0.50$ NA objectives, an AxioCamMRm
CCD camera (Carl Zeiss), and Axiovision3 imaging software (Carl Zeiss). Pseudo-color images of the ratio of Rab33a immunoreactivity to CMFDA staining and line-scan data were obtained using MetaMorph software (MDS Analytical Technologies). The relative fluorescence intensity of synaptophysin immunoreactivity and neurite length were analyzed with Multi Gauge software (Fujifilm). Time-lapse microscopy was performed at $37^{\circ} \mathrm{C}$ using a fluorescence microscope (IX81N-ZDC; Olympus) equipped with a CCD camera (C4742-80-12AG; Hamamatsu). Live-cell confocal fluorescence microscopy was performed at $37^{\circ} \mathrm{C}$ using a fluorescence microscope (Axio Observer Z1; Carl Zeiss) equipped with an $\alpha$ Plan-Apochromat 100× oil, 1.46 NA objective, an LSM 710 scan module (Carl Zeiss), and imaging software (ZEN2009; Carl Zeiss). Total internal reflection fluorescence (TIRF) microscopy was performed at $37^{\circ} \mathrm{C}$ using a fluorescence microscope (ECLIPSE TE2000-U; Nikon) equipped with an Apo TIRF 60× oil, 1.49 NA objective (Nikon) and a CCD camera (C9100; Hamamatsu). The acquired images were analyzed with Image (NIH).

Analysis of guanine nucleotides bound to Rab33a proteins. Guanine nucleotides bound to GTP-binding proteins were analyzed as described previously (Satoh and Kaziro, 1995). Briefly, HEK293T cells expressing Flag-Rab33a proteins were metabolically radiolabeled with ${ }^{32} \mathrm{P}_{\mathrm{i}}(1.2$ $\mathrm{mCi} /$ dish) for $3 \mathrm{~h}$ in phosphate-free DMEM, and Flag-Rab33a proteins were immunoprecipitated with an anti-FLAG M2 monoclonal antibody. Guanine nucleotides bound to Flag-Rab33a proteins were separated by thin-layer chromatography.

Materials. Anti-Myc, tau-1, anti-synaptophysin, anti-MAP-2, antiGFP, anti-giantin, and anti-Trk antibodies were obtained from MBL, Millipore Bioscience Research Reagents, Progen, Sigma-Aldrich, MBL, Abcam, and Santa Cruz Biotechnology, respectively. Anti-TGN38, antiGM130, and anti-adaptin $\gamma$ antibodies were obtained from BD Transduction Laboratories. Anti-Rab4, Rab5, Rab8, and Rab11 antibodies were obtained from BD Transduction Laboratories. Anti-Rab7, Rab9 and Rab10 antibodies were obtained from Abcam. Rabbit polyclonal antibody against Rab33a was produced as reported previously (Tsuboi and Fukuda, 2006). Anti-actin antibody was obtained from Millipore Bioscience Research Reagents. The plasmid 24478: CMV::SypHy A4 was purchased from Addgene.

Statistical analysis. Significance was determined by unpaired Student's $t$ tests in most cases. For multiple comparisons in Figure $8 C$, we used one-way ANOVA with Schaffer's post hoc test. In the statistical analysis for Figure $7 B$, neurons with one neurite longer than $100 \mu \mathrm{m}$ and over twice as long as any other neurite were counted as neurons with an axon (stage 3 neurons; neurons with multiple axons were excluded; Mori et al., 2007).

\section{Results}

\section{Rab33a is upregulated during axon outgrowth and} preferentially accumulates in axons

To analyze the functions of Rab33a in neurons, we first examined its tissue and intracellular distribution. Consistent with previous reports (Zheng et al., 1997; Cheng et al., 2006; Lee et al., 2006), immunoblot analysis showed that Rab33a is expressed in the rat brain (Fig. $1 \mathrm{~A}$ ). On the other hand, it was undetectable in peripheral tissues including heart, liver, spleen, kidney, lung, skeletal muscle, and placenta. Rab33a expression peaked around E18 in the developing brain, and decreased moderately after postnatal day 7 (P7; Fig. 1 B). The processes of axon and dendrite formation have been extensively studied using cultured rat hippocampal neurons (Craig and Banker, 1994). They first form several immature minor processes during the first $12-24 \mathrm{~h}$ after plating (stage 2). After $0.5-1.5 \mathrm{~d}$ in vitro (DIV), one of the neurites extends rapidly to become an axon (stage 3 ), while the others later become dendrites (stage 4, DIV4). The level of Rab33a expression increased during the transition from stage 2 to stage 3 in these neurons, and peaked on DIV7 (Fig. 1C). Thereafter, Rab33a expression returned to a relatively low level by DIV14, when neuronal networks become established in culture (stage 5). Thus, both in the brain and in cultured hippocampal neurons, the ex- 
A

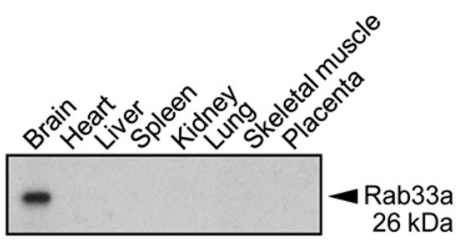

B

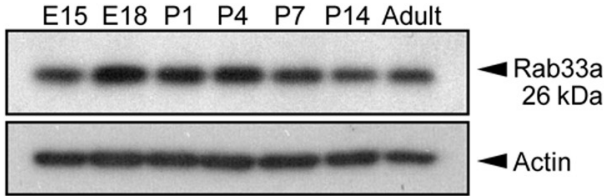

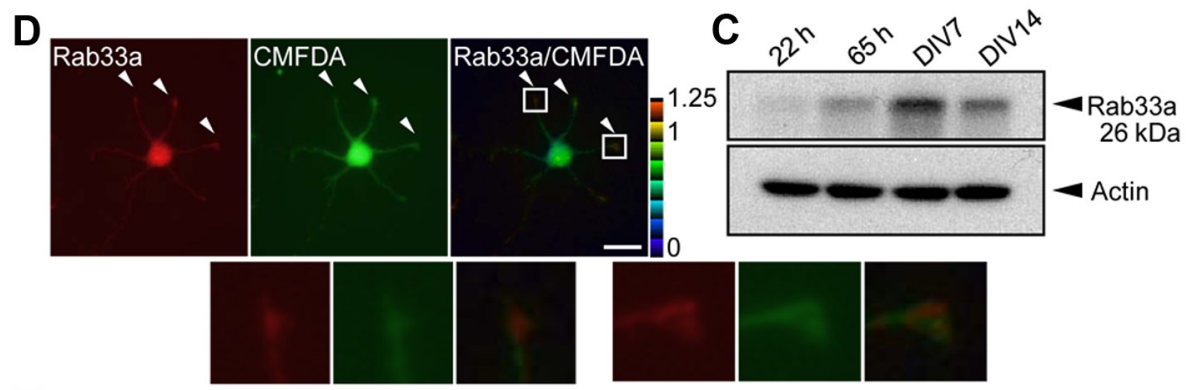
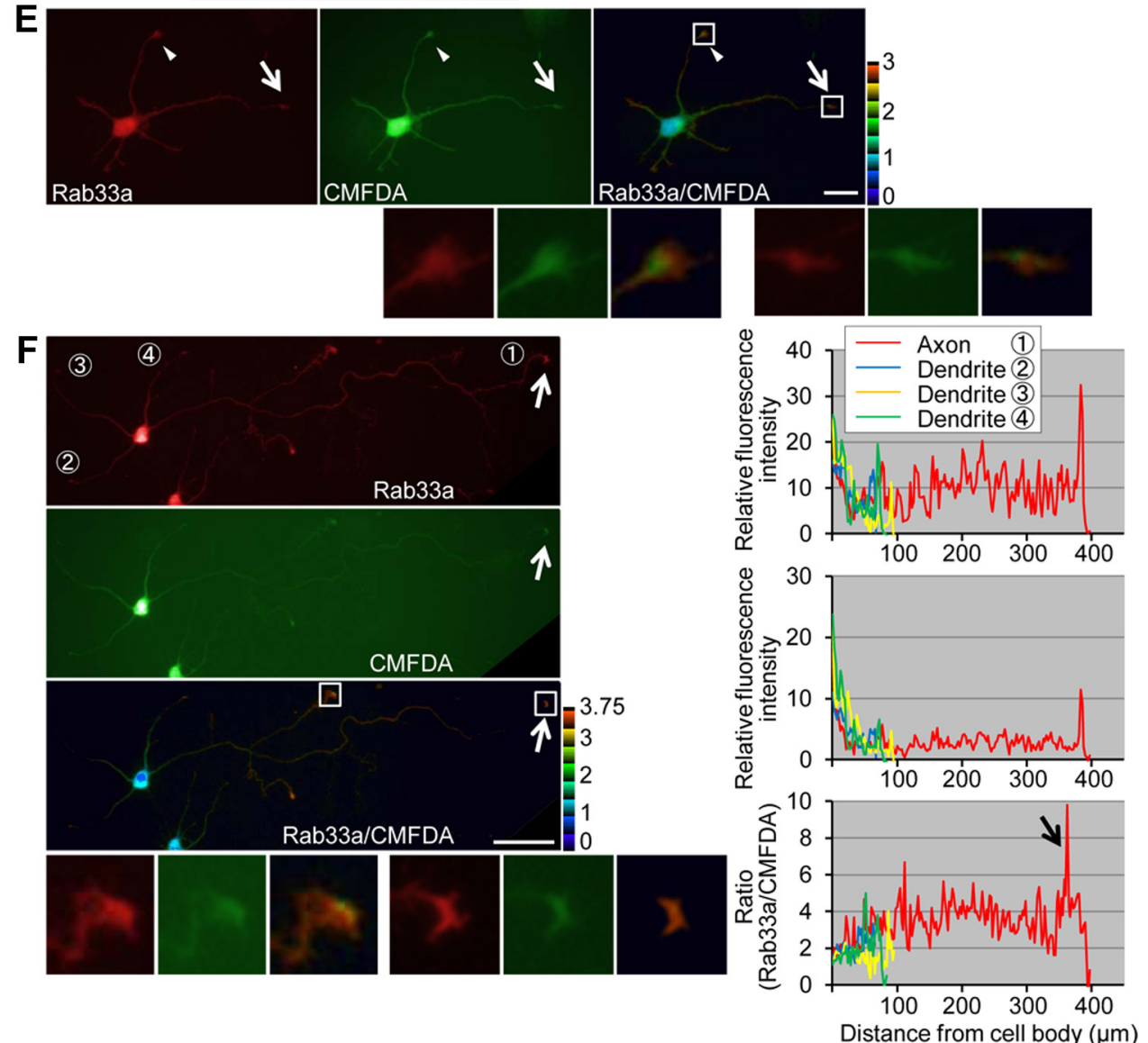

Figure 1. Expression and intracellular localization of Rab33a. $\boldsymbol{A}$, Immunoblot analysis of Rab33a in adult rat tissues. $\boldsymbol{B}, \boldsymbol{C}$, Immunoblot analysis of Rab33a in developing rat brain (B) and in rat cultured hippocampal neurons at stage 2 (cultured for $22 \mathrm{~h}$ ), stage 3 (cultured for 65 h), stage 4 (cultured for $7 \mathrm{~d}$ ), and stage 5 (cultured for $14 \mathrm{~d}$ ) (C). Immunoblots with anti-actin antibody served as loading controls. $\boldsymbol{D}-\boldsymbol{F}$, Immunofluorescence localization of Rab33a in stage $2(\boldsymbol{D})$, stage $3(\boldsymbol{E})$, and stage $4(\boldsymbol{F})$ hippocampal neurons. Neurons were double-stained by anti-Rab33a antibody (red) and the volume marker CMFDA (green). Pseudo-color images show the relative concentration of Rab33a (Rab33a immunoreactivity/CMFDA staining). Quantitative profiles show the relative fluorescence intensities of Rab33a (top) and CMFDA (middle) and the relative concentration of Rab33a (bottom). Arrows and arrowheads denote axonal growth cones and minor process growth cones, respectively. Insets in $\boldsymbol{D}-\boldsymbol{F}$ show enlarged views of the growth cone in the rectangles. Scale bars: $\boldsymbol{D}, \boldsymbol{E}, 20 \mu \mathrm{m} ; \boldsymbol{F}, 50 \mu \mathrm{m}$.

pression of Rab33a is upregulated during the period of axon formation and outgrowth.

Immunocytochemical analysis of cultured hippocampal neurons showed a widespread distribution of endogenous Rab33a in stage $2-4$ neurons (Fig. $1 D-F$ ). To examine the relative accumulation of Rab33a, we used the green volume marker CMFDA as an internal standard (Rab33a immunoreactivity/CMFDA staining; Toriyama et al., 2006). In stage 2 , Rab33a immunoreactivity showed a moderate accumulation in the growth cones of minor processes (Fig. $1 D$, arrowheads). In stage 3, Rab33a accumulated in the growth cones of axons as well as of minor processes (Fig. $1 E$, arrows and arrowheads). Stage 4 neurons showed remarkably more Rab33a accumulation in axons (Fig. $1 F$, arrows): Rab33a immunoreactivity was detected in the cell bodies and dendrites, but the relative Rab33a accumulation in axonal growth cones and shafts was markedly higher than in these regions. 


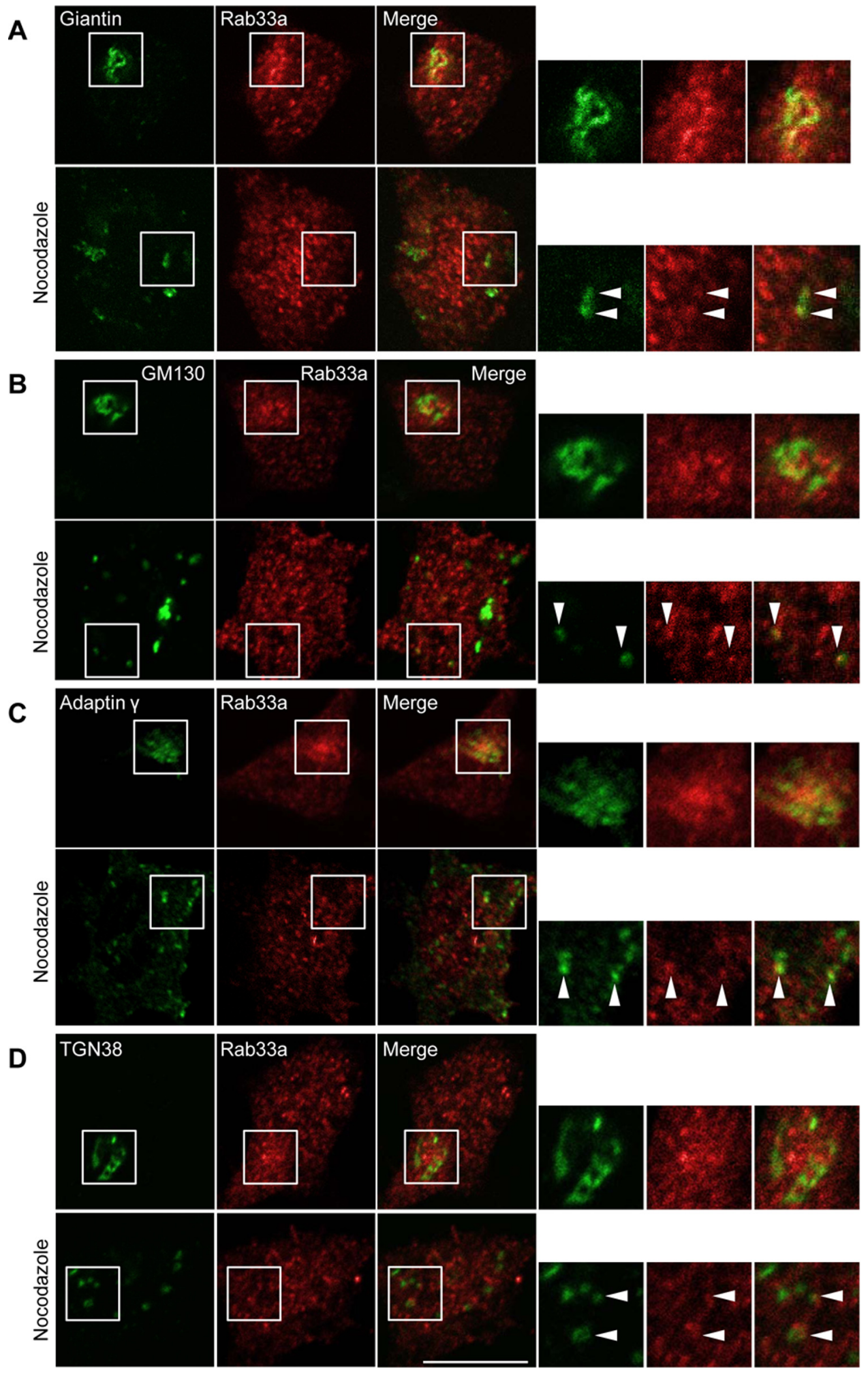

Figure 2. Rab33a is localized to the Golgi apparatus. $\boldsymbol{A}-\boldsymbol{D}$, Immunofluorescence localization of Rab33a and the Golgi markers giantin $(\boldsymbol{A})$, GM130 (B), adaptin $\gamma(\boldsymbol{C})$, and TGN38 (D) in the cell bodies of hippocampal neurons under control (top) or nocodazole (bottom) treatment. Arrowheads indicate colocalization of Rab33a with the Golgi markers. Scale bar, $10 \mu \mathrm{m}$.

Rab33a is localized to the Golgi apparatus and synaptophysin-positive vesicles transported along axons We further analyzed the subcellular localization of Rab33a in hippocampal neurons. Rab33a showed punctate and widespread distribution in the cell body. A part of Rab33a-immunoreactive puncta accumulated in the intracellular region where the Golgi markers giantin (Linstedt and Hauri, 1993), GM130 (Nakamura et al., 1995), adaptin $\gamma$ (Robinson, 1990), and TGN38 (Luzio et al., 1990) were localized (Fig. 2A-D, top). To confirm Golgi localization of these Rab33a, we treated the neurons with nocodazole, which induces the formation of highly polarized Golgi ministacks (Rogalski and Singer, 1984). Incubation of the neu- 


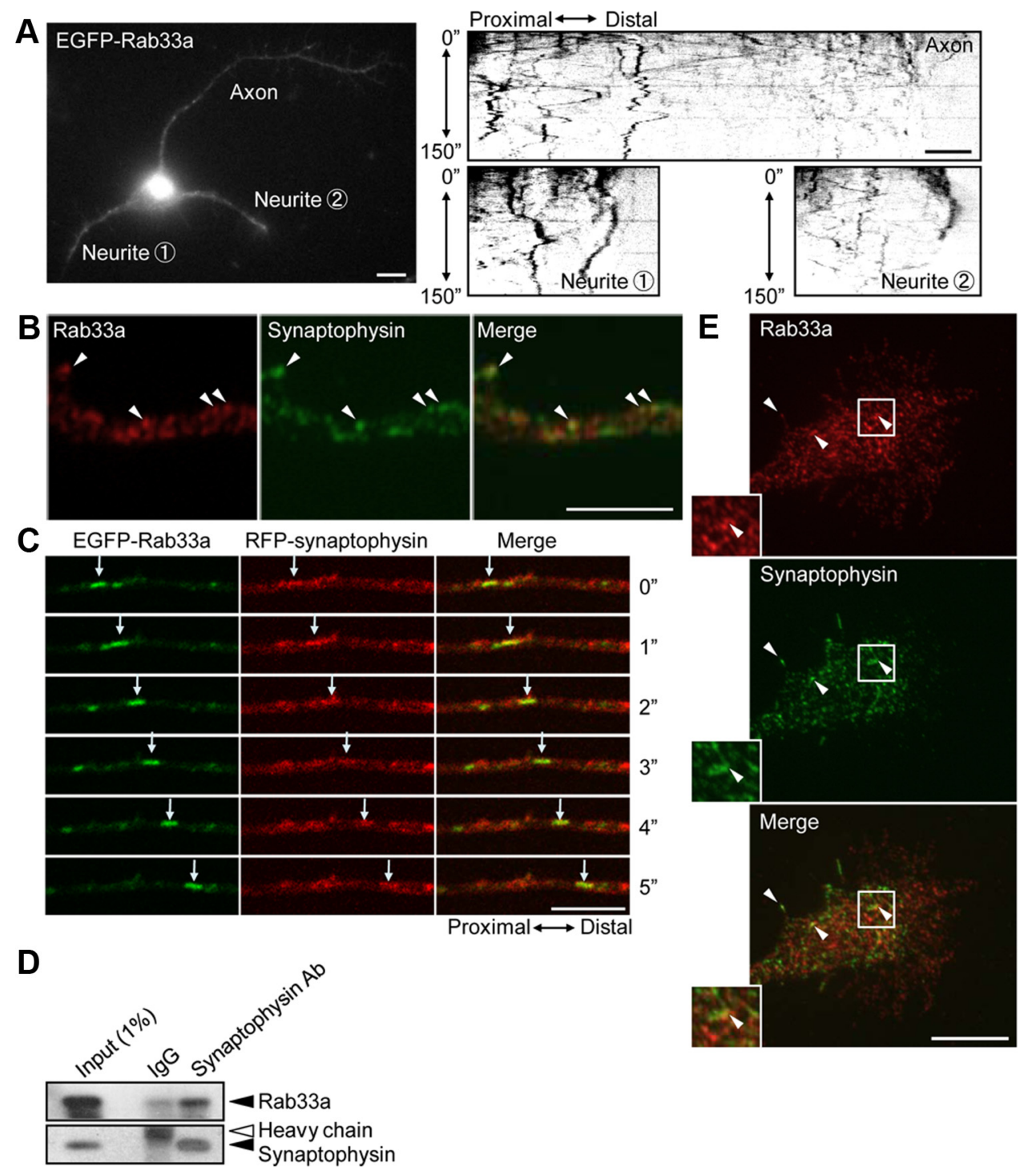

Figure 3. Rab33a is localized to synaptophysin-positive vesicles transported along axons. A, A stage 3 hippocampal neuron expressing EGFP-Rab33a was observed under a time-lapse fluorescence microscope every $1 \mathrm{~s}$ (left). The kymographs (right) of the three neurites show anterograde and retrograde transport of EGFP-Rab33a-positive vesicles. Images were captured every $1 \mathrm{~s}$ for $150 \mathrm{~s}$. $\boldsymbol{B}, \boldsymbol{E}$, Immunofluorescence localization of Rab33a (red) and synaptophysin (green) in an axon shaft ( $\boldsymbol{B})$ and a growth cone $(\boldsymbol{E})$ of a cultured hippocampal neuron. Arrowheads denote colocalization of Rab33a and synaptophysin. C, Cotransport of EGFP-Rab33a with RFP-synaptophysin in an axon of a hippocampal neuron observed by confocal microscopy. A vesicle bearing both EGFP-Rab33a and RFP-synaptophysin is indicated by arrows. D, Copurification of Rab33a with synaptophysin-containing vesicles of cultured hippocampal neurons. Immunoaffinity purification of synaptophysincontaining vesicles was performed, and the purified fractions were analyzed by immunoblot with anti-Rab33a antibody. Scale bars: $A, 10 \mu \mathrm{m} ; \boldsymbol{B}, C, E, 5 \mu \mathrm{m}$.

rons with $7 \mu \mathrm{M}$ nocodazole induced formation of Golgi ministacks dispersed throughout the cell bodies (Fig. $2 A-D$, bottom). This treatment led to a similar dispersal of Rab33a localization throughout the cell bodies. As shown in Figure 2, the dispersed Rab33a immunoreactivity colocalized partially with each of the dispersed Golgi markers: $14 \pm 2.5 \%$ of the giantin-positive area overlapped with the Rab33a-positive area; $9.0 \pm 2.1 \%$ of the GM130-positive area overlapped with the Rab33a-positive area; $12 \pm 2.0 \%$ of the adaptin $\gamma$-positive area overlapped with the Rab33a-positive area; and $8.9 \pm 1.8 \%$ of the TGN38-positive area overlapped with the Rab33a-positive area. Together, these data suggest that a subpopulation of Rab33a in the cell body is localized to the Golgi apparatus. As Rab family proteins play a key role in membrane trafficking, we monitored the movement of EGFPRab33a by live cell imaging. EGFP-Rab33a-positive puncta were observed in the soma; they were mobile and likely represented intracellular vesicular transport (Fig. 3A).
EGFP-Rab33a-containing vesicles also moved dynamically in neurites, in both anterograde and retrograde directions (Fig. $3 A$, kymographs). As shown in Figure 3B, Rab33a partially colocalized with synaptophysin-positive vesicles in axonal shafts (25.0 \pm $1.9 \%, n=399$ vesicles). Live imaging of hippocampal neurons expressing EGFP-Rab33a and red fluorescent protein (RFP)synaptophysin revealed that EGFP-Rab33a was cotransported with RFP-synaptophysin along axons (Fig. 3C). To confirm that synaptophysin and Rab33a are present on the same secretory vesicles, synaptophysin-containing vesicles were affinity-purified with anti-synaptophysin-coated beads. As shown in Figure $3 D$, Rab33a was copurified with synaptophysin-containing vesicles. Rab33a also localized to synaptophysin-positive vesicles in axonal growth cones, but the degree of colocalization was lower than that in the axonal shafts $(8.0 \pm 0.5 \%, n=814$ vesicles; Fig. $3 E$, arrowheads). Synaptophysin is a vesicular transmembrane protein that is sorted at the Golgi apparatus into the exocytic 


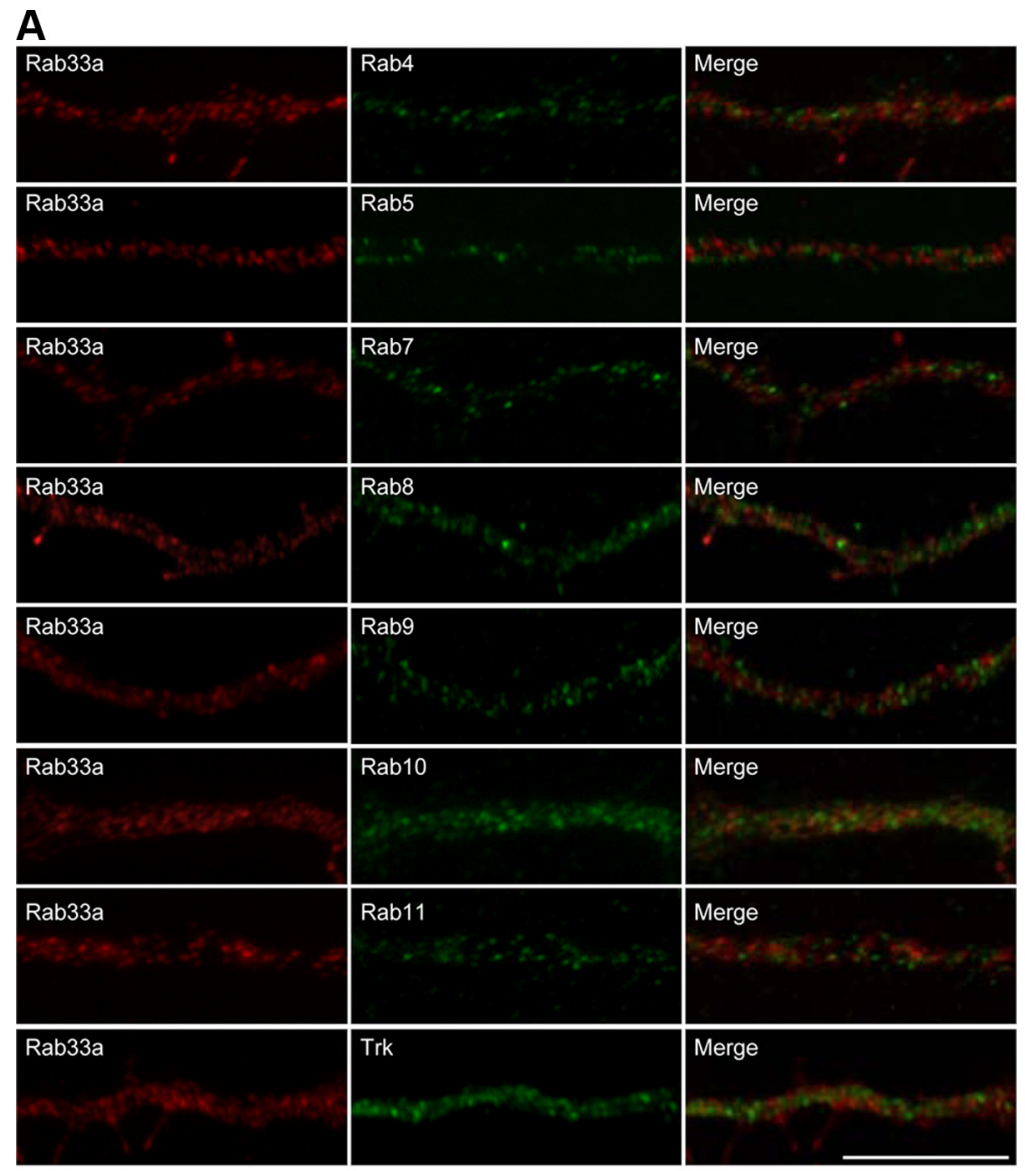

B

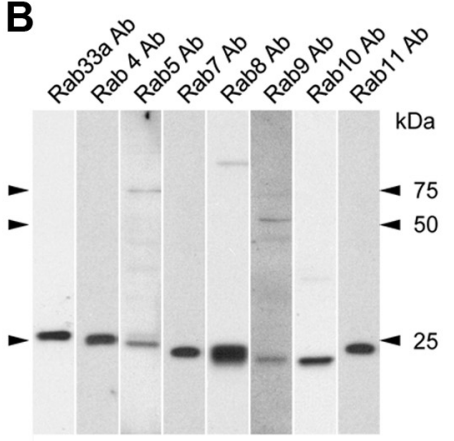

Figure 4. Subcellular localization of Rab33a in axons of cultured hippocampal neurons. $\boldsymbol{A}$, Immunofluorescence localization of Rab33a (red) and early endosome markers Rab4, Rab5, and Rab10, late endosome markers Rab7 and Rab9, recycling endosome markers Rab8 and Rab11, and Trk (green). Note the distinct localizations of Rab33a and these proteins. B, Immunoblot analyses to test the specificity of the antibodies used in $\boldsymbol{A}$. All antibodies recognized major bands at the correct molecular weights of the target molecules, thereby suggesting that the antibodies recognize their antigens. The anti-Rab5, Rab8, and Rab9 antibodies recognized faint bands at $\sim 75,90$, and $50 \mathrm{kDa}$, respectively, in addition to the main band, indicating that they interact with additional unknown molecules. However, since the data in $\boldsymbol{A}$ show that the immunoreactivity of anti-Rab5, Rab8, and Rab9 antibodies does not overlap with that of anti-Rab33a antibody, Rab33a is unlikely to colocalize with Rab5, Rab8, Rab9, or the unknown molecules in the axons. Scale bar, $10 \mu \mathrm{m}$.

pathway and transported by fast axonal transport to the plasma membrane at nerve terminals, where it cycles between the plasma membrane and endosomes (De Camilli and Jahn, 1990; Kelly, 1991; Régnier-Vigouroux et al., 1991; Nakata et al., 1998; Kaether et al., 2000). Before the formation of synaptic contacts, it also accumulates in distal axons and growth cones of developing neurons (Fletcher et al., 1991; Phelan and Gordon-Weeks, 1992).

To determine whether Rab33a is localized to post-Golgi synaptophysin-positive vesicles or to endocytic synaptophysinpositive vesicles, we performed double immunostaining for Rab33a and endocytic Rabs. As shown in Figure 4A, Rab33a did not colocal- ize with endocytic Rabs: Rab4, Rab5, and Rab10 located to early endosomes (van der Sluijs et al., 1992; Sönnichsen et al., 2000; Babbey et al., 2006; Deinhardt et al., 2006), Rab7 and Rab9 located to late endosomes (Lombardi et al., 1993; Deinhardt et al., 2006), and Rab8 and Rab11 located to recycling endosomes (Ullrich et al., 1996; Sönnichsen et al., 2000; Hattula et al., 2006; Roland et al., 2007; Ascaño et al., 2009); immunoblot data for the ant-Rab antibodies are shown in Figure $4 B$. This is consistent with a previous report that Rab33a localizes to secretory vesicles in PC12 cells (Tsuboi and Fukuda, 2006). Rab33a also did not localize to Trk-positive vesicles, which are reported to be transported by Rab27 and Rab11 (Arimura et al., 2009; Ascaño et al., 2009). Given its accumulation in the Golgi apparatus (Fig. 2), these results suggest that Rab33a is most likely associated with the post-Golgi synaptophysin-positive vesicles that are transported along axons.

\section{Reduction of Rab33a expression inhibits anterograde axonal transport of synaptophysin-positive vesicles} Next, we analyzed the involvement of Rab33a in axonal vesicular transport using synaptophysin as a vesicle marker: vesicles that moved along proximal regions of axons (200 $\mu \mathrm{m}$ length) adjacent to the soma were monitored (Fig. 5A). In agreement with previous reports (Nakata et al., 1998; Kaether et al., 2000), vesicles labeled by RFP-synaptophysin moved in both anterograde and retrograde directions (Fig. 5A, kymograph). They often switched their direction of movement, and some were immobile during the observation period. We categorized the patterns of their movement during $20 \mathrm{~s}$ observations ( $0.1 \mathrm{~s}$ interval) into three groups: (1) vesicles that showed anterograde net movement (anterograde), (2) vesicles that showed retrograde net movement (retrograde), (3) and those did not show detectable net movement (immobile).

Rab33a expression was suppressed using a vector-based RNAi system that expresses miRNA against Rab33a. A reduction of endogenous Rab33a in hippocampal neurons by two miRNAs (\#1 and \#2) was confirmed by immunoblot (Fig. $5 B$ ) and immunocytochemical (Fig. 5C) analyses. Knockdown of Rab33a significantly decreased the number of total (Fig. 5D) and anterograde and immobile RFP-synaptophysin-positive vesicles observed in the regions analyzed (Fig. $5 E$ ). On the other hand, the number of retrograde vesicles did not change significantly following Rab33a RNAi. Figure 5, F and $G$, shows further analysis of the velocity of the moving vesicles labeled by RFP-synaptophysin. In neurons expressing a control miRNA, the mean velocities of the anterograde and retrograde movement were $3.5 \pm 0.4 \mu \mathrm{m} / \mathrm{s}$ and $2.5 \pm 0.3 \mu \mathrm{m} / \mathrm{s}$, respectively (Fig. $5 F$ ). Suppression of Rab33a expression by miRNAs 
A
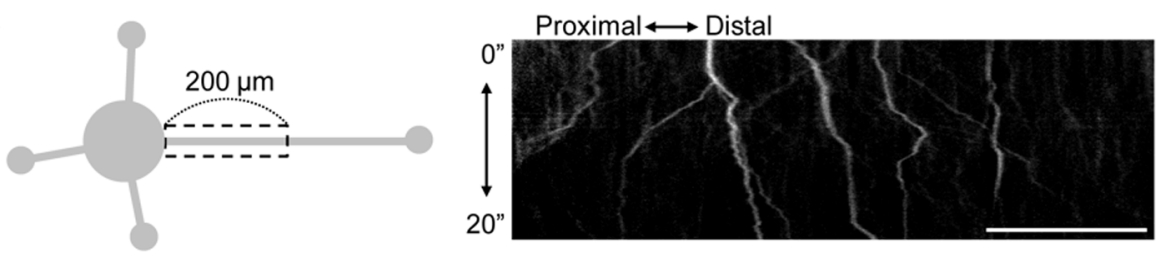

B
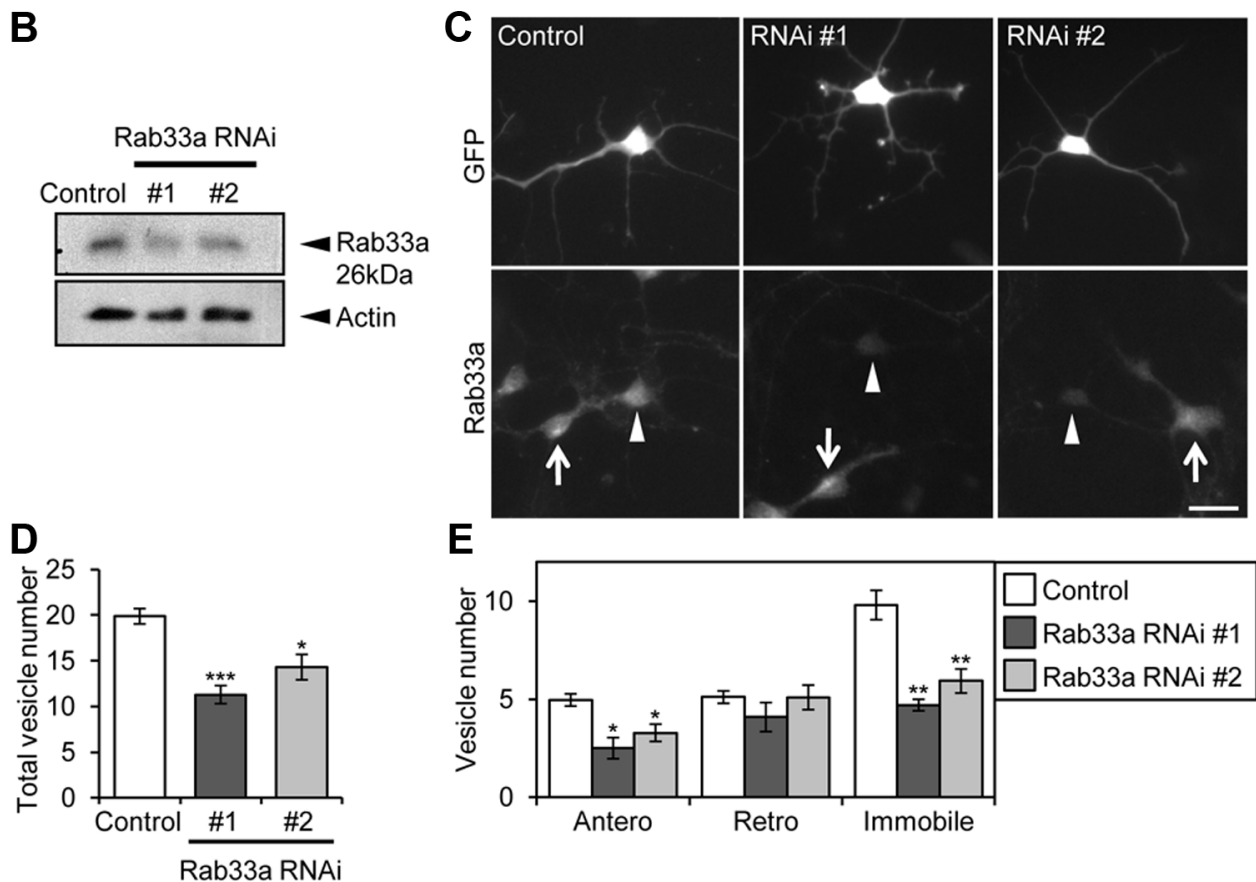

$\mathbf{E}$
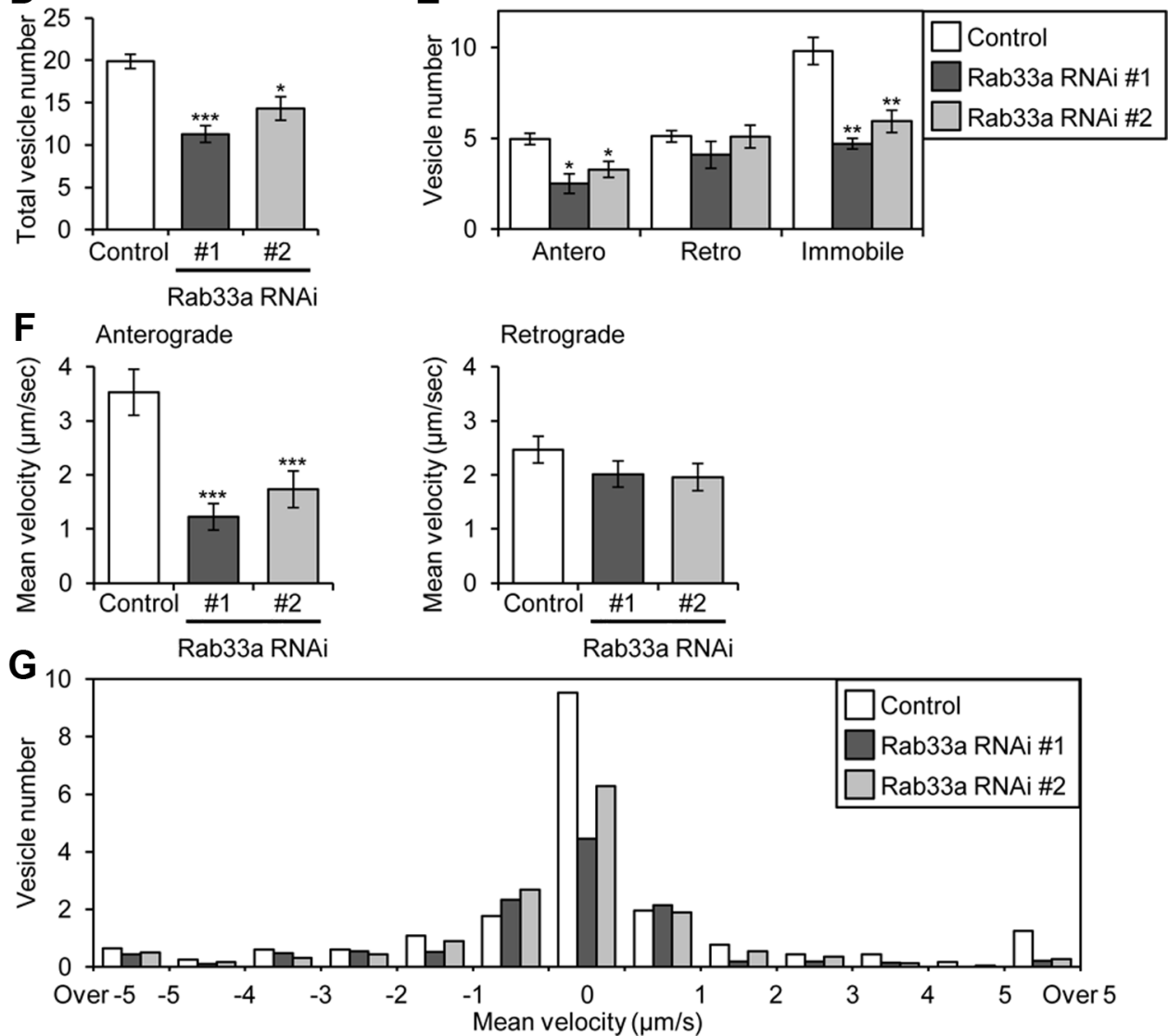

Figure 5. Rab33a is involved in the anterograde axonal transport of synaptophysin-positive vesicles. $A$, A schematic illustration of the region of observation of mRFP-synaptophysin-positive vesicles (left) and a kymograph of the transport (right). Images were captured every $100 \mathrm{~ms}$ for $20 \mathrm{sin}$ an axon. B, Suppression of Rab33a by miRNAi in hippocampal neurons. Proteins extracted from hippocampal neurons expressing control miRNA or miRNA against Rab33a (\#1 or \#2) were subjected to SDS-PAGE and the amount of Rab33a in each sample was examined by immunoblot. C, Rab33a immunoreactivity of neurons transfected with control miRNA or miRNA against Rab33a (\#1 or \#2). Neurons were fixed at $36 \mathrm{~h}$ in culture. The immunoreactivity of Rab33a in GFP-positive neurons was compared with that of control miRNA neurons. The arrowheads and arrows indicate GFP-positive transfected and untransfected neurons, respectively. $\boldsymbol{D}$, Total vesicle number observed in the boxed region in $\boldsymbol{A}$. Error bars indicate SEM. ${ }^{*} p<0.05$ and ${ }^{* * *} p<0.005$ relative to control, Student's $t$ tests. $\boldsymbol{E}$, The number of anterograde, retrograde, and immobile vesicles observed in the region in $\boldsymbol{A}$. Error bars indicate SEM. ${ }^{*} p<0.05$ and ${ }^{* *} p<0.01$ relative to control, Student's $t$ tests. $F$, Mean velocities of anterograde (left) and retrograde (right) transport of mRFP-synaptophysin-positive vesicles. Error bars indicate SEM. ${ }^{* * *} p<0.005$ relative to control, Student's $t$ tests. $G$, The number of vesicles with indicated velocities observed in the region in $A$. A total of 450 vesicles in 23 neurons for control miRNA, 318 vesicles in 27 neurons for Rab33a miRNA \#1, and 321 vesicles in 22 neurons for Rab33a miRNA \#2 were examined in four independent experiments. Scale bars: $A, 50 \mu \mathrm{m}$; $C$, $20 \mu \mathrm{m}$. 

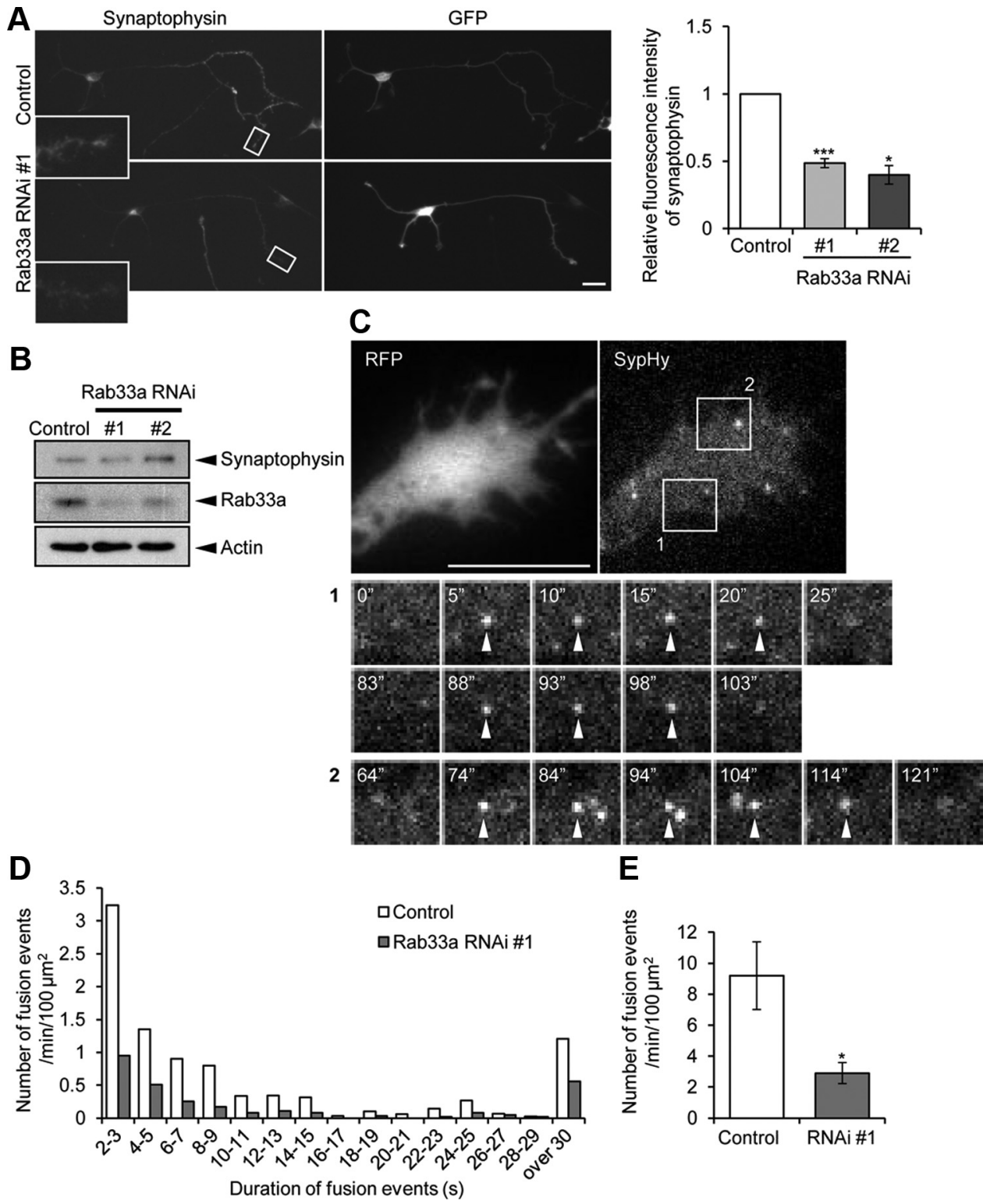

E

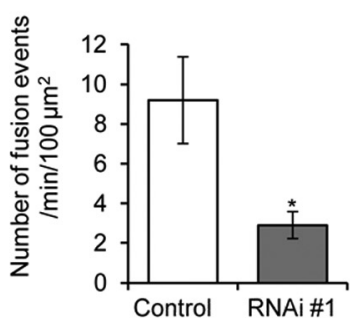

Figure 6. Rab33a is involved in fusion of synaptophysin-positive vesicles at the growth cone. $A$, Synaptophysin immunoreactivity of neurons transfected with control miRNA or miRNA against Rab33a. Insets in $A$ show enlarged views of the growth cones in the rectangles. Relative fluorescence intensity of synaptophysin in the distal region of the axon ( $20 \mu \mathrm{m}$ length, rectangles) was quantified. A total of 108 neurons for control miRNA, 104 neurons for Rab33a miRNA\#1, and 89 neurons for Rab33a miRNA\#2 were examined in three independent experiments. Error bars indicate SEM. ${ }^{*} p<0.05$ and ${ }^{* * *} p<0.005$ relative to control, Student's t tests. $\boldsymbol{B}$, Immunoblot analyses of endogenous synaptophysin and Rab33a in cultured neurons transfected with control miRNA, miRNA against Rab33a \#1, or miRNA against Rab33a \#2. C-E, Hippocampal neurons were transfected with SypHy and observed using TIRF microscopy. C, A growth cone of a hippocampal neuron expressing RFP and SypHy (top). Representative images from a time-lapse series (bottom) display vesicle fusion events at the indicated areas 1 and 2 of the growth cone (arrowheads). D, Histogram of the duration of SypHy signals in growth cones cotransfected with SypHy and Rab33a miRNA\#1 or control miRNA. E, Frequency of vesicle fusion in the growth cones was quantified. A total of 227 vesicles in 12 growth cones for control miRNA and 111 vesicles in 11 growth cones for Rab33a miRNA $\# 1$ were examined. Error bars indicate SEM. ${ }^{*} p<0.05$ relative to control, Student's $t$ tests. Scale bars: $A, 20 \mu \mathrm{m} ; \boldsymbol{C}, 10 \mu \mathrm{m}$.

\#1 or \#2 remarkably decreased the mean velocity of anterograde movement. Histogram analysis of the velocity shows that fast components of the transport $(>1 \mu \mathrm{m} / \mathrm{s}$ ) were reduced (Fig. $5 G$ ). In contrast, no significant differences were observed in the mean velocity of the retrograde transport (Fig. 5F). The reduction of both the number and the velocity of the anterogradely moving vesicles thus suggest that Rab33a is involved in anterograde transport of synaptophysin-positive vesicles.

Reduction of Rab33a expression inhibits membrane fusion of synaptophysin-positive vesicles at the growth cones

Next, we examined the possible role of Rab33a in axonal membrane insertion. Endogenous synaptophysin was preferentially localized in growing axons of control neurons, as reported previously (Fletcher et al., 1991; Phelan and Gordon-Weeks, 1992) (Fig. 6A). In agreement with the above-mentioned data demonstrating the involvement of Rab33a in anterograde transport of synaptophysin-positive vesicles, Rab33a RNAi led to a significant reduction of endogenous synaptophysin intensity in the distal regions of axons (Fig. $6 A$, rectangles). The total amount of synaptophysin in hippocampal neurons was not affected by Rab33a RNAi (Fig. 6B).

The reduction of synaptophysin level in distal axons by Rab33a RNAi led us to further examine the correlation between the Rab33a level and the insertion of synaptophysinpositive vesicles at the growth cones. We used synaptophysin 

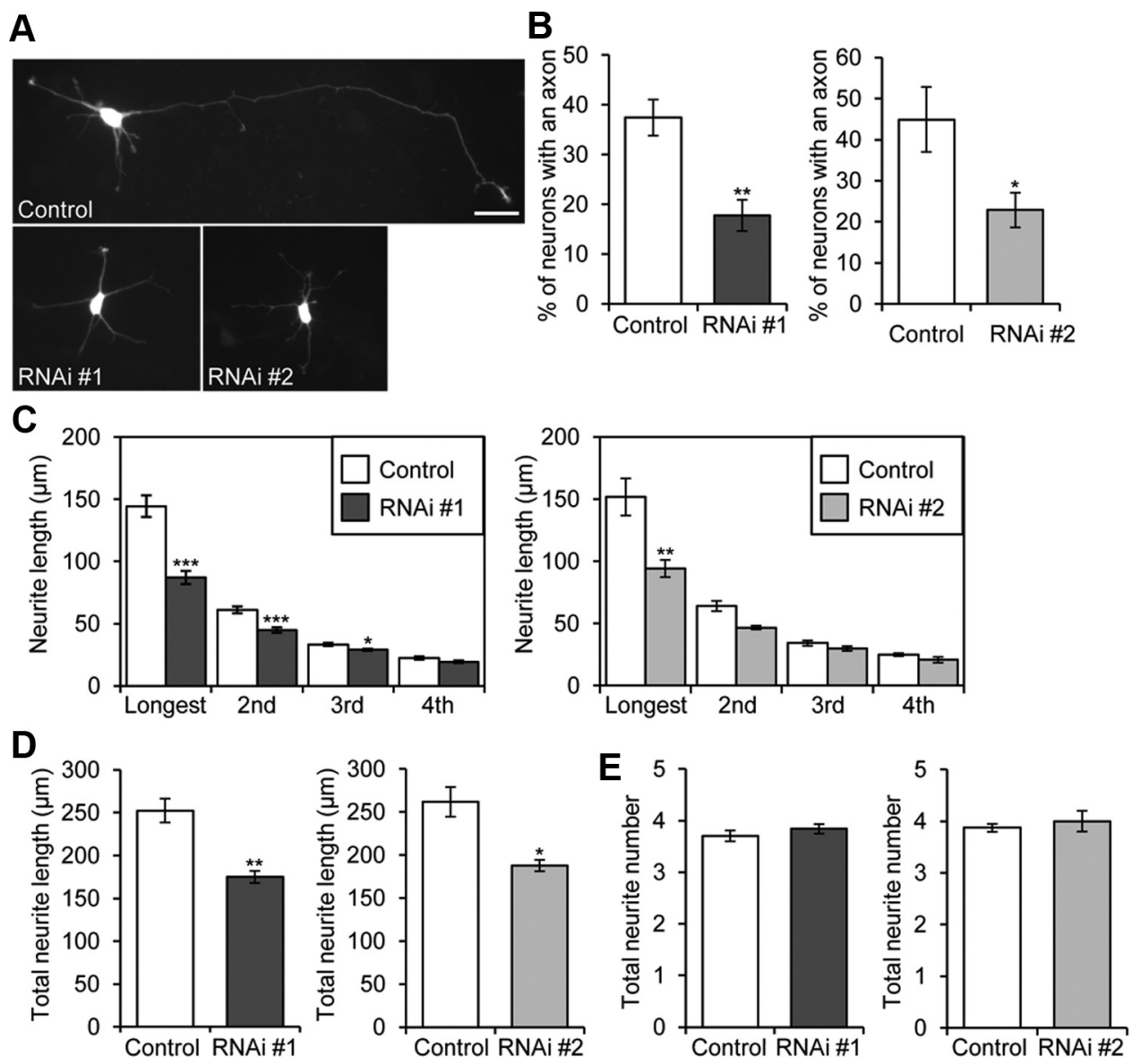

Figure 7. Suppression of Rab33a expression inhibits axon formation and axon outgrowth. $A-E$, Morphological analyses of Rab33a RNAi-treated neurons. $A$, Neurons visualized by the fluorescence of GFP. B, Percentage of neurons with an axon. $\boldsymbol{C}$, Neurite length of Rab33a RNAi-treated neurons. D, Total neurite length. $\boldsymbol{E}$, Total neurite number. Neurons transfected with control miRNA, Rab33a miRNA \#1, or Rab33a miRNA \#2 were fixed at $36 \mathrm{~h}$ in culture and immunostained by anti-Rab33a and anti-GFP antibodies. In the analyses with miRNA \#1, a total of 386 neurons for control and 332 neurons for Rab33a RNAi \#1 were examined in six independent experiments. In the analyses with miRNA \#2, a total of 276 neurons for control and 238 neurons for Rab33a RNAi \#2 were examined in four independent experiments. Error bars indicate SEM. ${ }^{*} p<0.05,{ }^{* *} p<0.01,{ }^{* * *} p<0.005$ relative to control, Student's $t$ tests. Scale bar, $20 \mu \mathrm{m}$.

fused with a pH-sensitive GFP pHluorin (Miesenböck et al., 1998), namedSypHy, asamarkerofexocytosisofsynaptophysinpositive vesicles (Granseth et al., 2006). SypHy is quenched by the acidic $\mathrm{pH}$ inside a vesicle, but fluorescence emission increases when the interior of the vesicle connects with the external medium upon exocytosis. Membrane fusion was observed using a TIRF microscope, which allows selective imaging of fluorescence signals located in close proximity $(<200$ $\mathrm{nm}$ ) to the coverslips (Wang et al., 2011). The TIRF signals of SypHy were observed mainly in the soma and distal region of axons and the growth cones, consistent with previous observations that membrane insertions occur mainly in the cell bodies and distal axons (Dupraz et al., 2009; Wang et al., 2011).

Figure $6 C$ shows representative fusion events in an axonal growth cone. Images were captured at $1 \mathrm{~s}$ intervals for $4 \mathrm{~min}$. To exclude vesicles that were not inserted into the plasma membrane or that stayed near the membrane, we only counted signals that remained immobile for at least $2 \mathrm{~s}$. The TIRF signals were observed widely in the growth cones, but located preferentially in the marginal region of the central domain of the growth cones. The duration of the SypHy signals varied widely, with a peak of $2-3 \mathrm{~s}$ (Fig. 6D). The mean frequency of membrane fusion of the SypHy-labeled vesicles in the growth cones was $9.2 \pm 2.2 / \mathrm{min} /$
$100 \mu \mathrm{m}^{2}$ (Fig. 6E). As shown in Figure 6, $D$ and $E$, reduction of Rab33a by RNAi led to a significant decrease in the frequency of fusion events in the growth cones.

\section{Reduction of Rab33a expression inhibits axon formation and outgrowth}

We further examined the effect of Rab33a RNAi on axon formation and outgrowth. Suppression of Rab33a expression led to significant inhibition in axon formation at $36 \mathrm{~h}$ in culture: compared with control neurons $(37 \pm 3.6 \%)$, only $18 \pm 3.1 \%$ of the neurons with Rab33a miRNA \#1 formed an axon (Fig. 7A,B), and similar results were obtained with Rab33a miRNA \#2. Rab33a knockdown by miRNA \#1 resulted in a $38.7 \%$ reduction in the length of the longest neurite (including the axon) and a $21.5 \%$ reduction in dendrite length, while knockdown by miRNA $\# 2$ resulted in a $38.6 \%$ reduction in axon length and a $17.7 \%$ reduction in dendrite length (Fig. 7C). Thus, we consider that Rab33a knockdown influences both axon and dendrite outgrowth; however, the effect on axon outgrowth was more marked. This is consistent with the preferential, but not exclusive, localization of Rab33a in axons (Fig. $1 F$ ). Suppression of Rab33a expression also led to a decrease in the total neurite length (Fig. $7 D)$. However, total neurite number in the neurons expressing Rab33a miRNAs was similar to that of control neurons (Fig. 7E). 

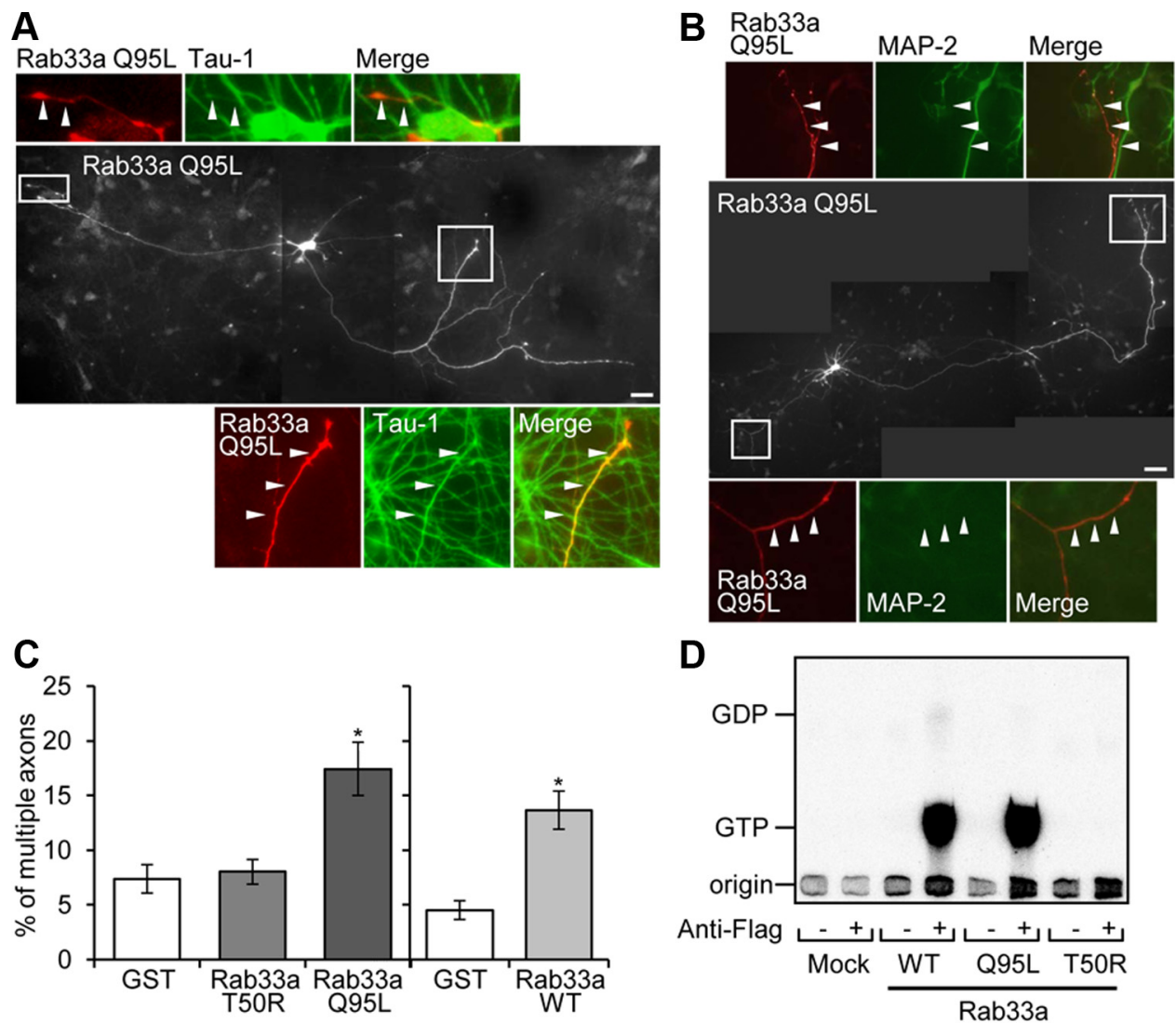

D
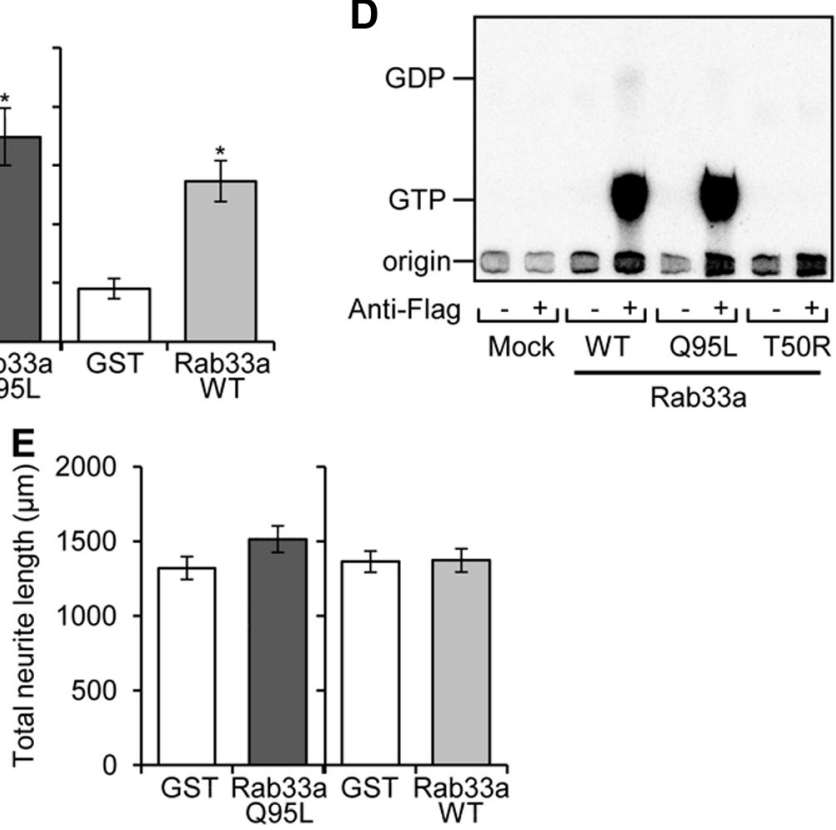

Figure 8. Overexpression of Rab33a induces formation of multiple axons. A, Hippocampal neurons overexpressing Myc-Rab33a Q95L were cultured for $6 \mathrm{~d}$ and then immunostained with anti-Myc (red) and tau- 1 (green) antibodies. Arrowheads in the enlargements of the two boxed areas of the main image indicate axons labeled by tau- 1 antibody. B, Hippocampal neurons overexpressing Myc-Rab33a Q95L were cultured for $6 \mathrm{~d}$ and then double immunostained with anti-Myc (red) and anti-MAP-2 (green) antibodies. Arrowheads in the enlarged images indicate axons unlabeled by anti-MAP-2 antibody. C, Percentage of neurons with multiple axons. Neurons transfected with Myc-GST, Myc-Rab33a Q95L, Myc-Rab33a T50R, or Myc-Rab33a WT were fixed on DIV6 and analyzed. A total of 127 neurons for GST, 120 neurons for Rab33a WT (right), 74 neurons for GST, 83 neurons for Rab33a T50R, and 76 neurons for Rab33a Q95L (left) were examined in three independent experiments. Error bars indicate SEM. ${ }^{*} p<0.05$ relative to GST or Rab33a T50R, one-way ANOVA analysis with Schaffer's post hoc test (left). ${ }^{*} p<0.05$ relative to GST, Student's $t$ tests (right). $\boldsymbol{D}$, Guanine nucleotide-bound form of WT and mutants of Rab33a expressed in HEK293T cells. HEK293T cells were transfected with expression vectors encoding the N-terminally FLAG-tagged Rab33a proteins indicated at the bottom and metabolically radiolabeled with ${ }^{32} \mathrm{P}_{\mathrm{i}}$. FLAG-Rab33a proteins were immunoprecipitated with an anti-FLAG M2 monoclonal antibody. Guanine nucleotides bound to Flag-Rab33a proteins were separated by thin-layer chromatography. E, Total neurite length of neurons overexpressing Myc-GST, Myc-Rab33a WT, or Myc-Rab33a Q95L on DIV6. Error bars indicate SEM. Student's $t$ tests. Scale bars: $\boldsymbol{A}, \boldsymbol{B}, 50 \mu \mathrm{m}$.

\section{Overexpression of functional Rab33a induces excessive accumulation of synaptophysin-positive vesicles and formation of surplus axons}

As previous studies report that, in many cases, overexpression of molecules involved in axon formation and outgrowth induces the formation of aberrant axons from minor processes (Inagaki et al., 2001; Kunda et al., 2001; Toriyama et al., 2006), we finally overexpressed a Myc-tagged constitutively active form of Rab33a, Myc-Rab33a Q95L. Remarkably, $17 \pm 2.4 \%$ ( $p<0.05$, compared with Myc-GST (glutathione $S$-transferase) or Myc-Rab33a T50R) of the neurons with overexpressed Myc-Rab33a Q95L bore multiple axons that were immunostained by the axon- specific marker tau- 1 antibody but were immunonegative for the dendrite-specific marker anti-MAP-2 antibody (Fig. $8 A-C$ ). In contrast, only $7.4 \pm 1.3 \%$ of control neurons with overexpressed Myc-GST formed supernumerary axons (Fig. 8C). Multiple axons were also induced by overexpressed Myc-tagged wild-type (WT) Rab33a but not by the inactive mutant Myc-Rab33a T50R (Fig. 8C). An in vitro experiment showed that WT Rab33a and Rab33a Q95L bound GTP, but that Rab33a T50R did not (Fig. $8 D$ ), thereby indicating a guanine nucleotide dependency of Rab33a-induced aberrant axon formation. The sum of the length of neurites in neurons overexpressing Rab33a WT or Rab33a Q95L was similar to that in control neurons on DIV6 (Fig. $8 E$ ). 


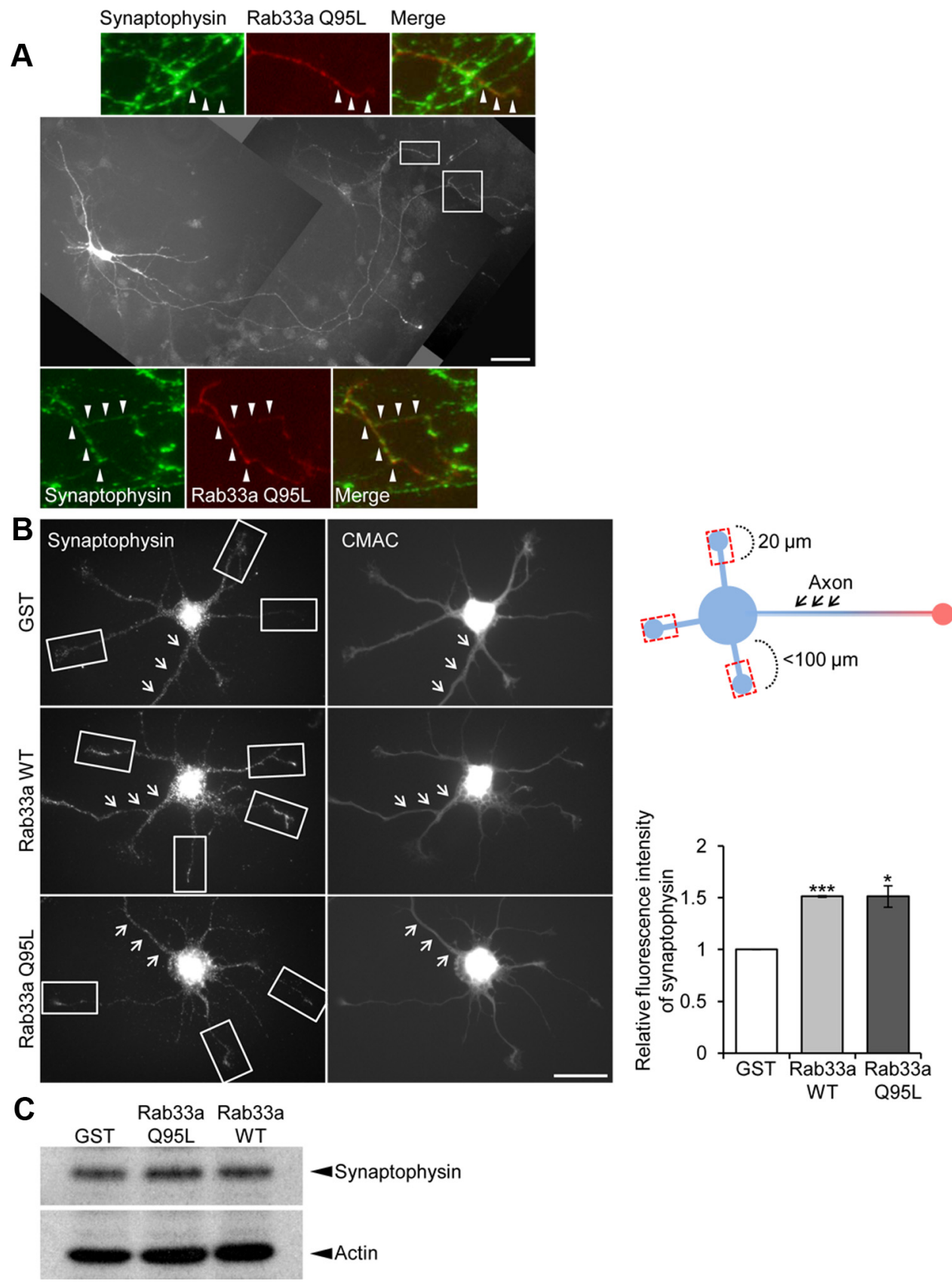

Figure 9. Overexpression of Rab33a induces excessive accumulation of synaptophysin-positive vesicles. A, Hippocampal neurons overexpressing Myc-Rab33a Q95L were cultured for $6 \mathrm{~d}$ and then immunostained with anti-Myc (red) and anti-synaptophysin (green) antibodies. Arrowheads in the enlargements of the two boxed areas of the main image indicate axons labeled by antisynaptophysin antibody. B, Synaptophysin immunoreactivity of short neurites $(<100 \mu \mathrm{m})$ of cultured hippocampal neurons overexpressing Myc-GST, Myc-Rab33a WT, or Myc-Rab33a Q95L (left). Neurons were fixed on DIV 3 and costained with the volume marker CMAC. Relative synaptophysin immunoreactivity in the distal region of minor processes ( $20 \mu \mathrm{m}$ length, rectangles) was quantified (right graph). Arrows indicate the axons. A total of 61 neurons for GST, 70 neurons for Rab33a WT, and 66 neurons for Rab33a QL were examined in three independent experiments. Error bars indicate SEM. ${ }^{*} p<0.05$ and ${ }^{* * *} p<0.005$ relative to GST, Student's $t$ tests. C, Immunoblot analysis of endogenous synaptophysin in cultured neurons overexpressing Myc-GST, Myc-Rab33a Q95L, or Myc-Rab33a WT. Scale bars: $A, 50 \mu \mathrm{m} ; \boldsymbol{B}, 20 \mu \mathrm{m}$.

We also examined the localization of endogenous synaptophysin in neurites of hippocampal neurons which overexpressed Rab33a. As shown in Figure 9A, the multiple axons induced by Rab33a Q95L overexpression were enriched in endogenous synaptophysin. This is consistent with previous observations that aberrant axons induced by overexpression of molecules involved in axon formation were enriched for synaptophysin (Inagaki et al., 2001; Toriyama et al., 2006; Mori et al., 2007). To exclude the possibility that the increase of endogenous synaptophysin signal could be due to induced formation of multiple axons rather than vesicular transport, we also quan- tified the intensity of synaptophysin immunoreactivity in the distal parts of minor processes (20 $\mu$ m length; Fig. $9 B$, rectangles). The intensity of endogenous synaptophysin in the minor processes of neurons overexpressing Rab33a (WT or Q95L) was $\sim 1.5$ times that in control neurons (Fig. 9B). The total amount of endogenous synaptophysin in hippocampal neurons was unaffected by Rab33a overexpression (Fig. 9C). Thus, the results from functional Rab33a overexpression experiments provide further evidence that Rab33a is involved in the transport of synaptophysin-positive vesicles and axon outgrowth. 


\section{Discussion}

Axon outgrowth is accompanied by plasma membrane expansion that results from membrane synthesis, vesicular transport and exocytosis (Bray, 1970; Lockerbie et al., 1991; Craig et al., 1995; Futerman and Banker, 1996; Pfenninger, 2009). We have shown that Rab33a expression became upregulated during axon outgrowth of cultured hippocampal neurons, where it localized to synaptophysin-positive vesicles that were transported along axons. The anterograde axonal transport of synaptophysinpositive vesicles was inhibited when Rab33a expression was reduced, leading to decreased numbers of vesicles in axonal tips. Repressing the Rab33a level further reduced both exocytosis of the synaptophysin-positive vesicles at the growth cones and axon outgrowth. In contrast, overexpression of Rab33a induced excessive accumulation of synaptophysin-positive vesicles, with a concurrent formation of supernumerary axons. Collectively, these results indicate a role for Rab33a in the anterograde transport of synaptophysin-positive vesicles, which is associated with their concomitant fusion at the growth cones and axon outgrowth.

Synaptophysin is a vesicular transmembrane protein transported from the Golgi apparatus through exocytic pathways to the plasma membrane at nerve terminals, where it cycles between the plasma membrane and early endosomes (De Camilli and Jahn, 1990; Kelly, 1991; Régnier-Vigouroux et al., 1991; Nakata et al., 1998) as a major component of synaptic vesicles (Jahn et al., 1985; Wiedenmann and Franke, 1985). Synaptophysin-positive vesicles are also exported from the Golgi apparatus and localized to growing axons before the formation of synaptic contacts (Fletcher et al., 1991; Phelan and Gordon-Weeks, 1992; Rosso et al., 2004). At the growth cones, synaptophysin is localized to vesicles which are thought be added to the plasma membrane (Saito et al., 1992; Igarashi et al., 1997). In this study, we directly demonstrated the insertion of synaptophysin-positive vesicles into the plasma membrane, by monitoring synaptophysin fused to a $\mathrm{pH}$-sensitive GFP with TIRF microscopy. Vesicular insertion occurred mainly in the marginal region of the central domain of the growth cones, where plasmalemmal precursor-like vesicles are observed (Pfenninger and Friedman, 1993; Pfenninger, 2009). Subsets of axonal proteins are known to be transcytosed from the somatodendritic membrane through endosomes to the axonal membrane (Hémar et al., 1997; Sampo et al., 2003; Wisco et al., 2003; Leterrier et al., 2006; McDonald et al., 2007; Winckler and Yap, 2011). However, Rab33 accumulated in the Golgi apparatus in the cell bodies, and did not colocalize with endosome markers in the axonal shafts. Thus, we consider that Rab33a is involved in the axonal transport of Golgi-derived vesicles, rather than endosomes.

How does Rab33a promote vesicular fusion at the growth cones? As Rab33a contributes to the anterograde transport of synaptophysin-positive vesicles, the increased supply of these vesicles from the cell body may indirectly upregulate their fusion events at the growth cones. The low degree of colocalization between Rab33a and synaptophysin observed in the growth cones supports this notion, although we cannot rule out the possibility that Rab33a directly regulates the insertion of synaptophysinpositive vesicles at the growth cones. Previous studies suggest that SNARE proteins, the TC10-Exo70 complex, and Rab10 are involved in vesicular fusion at the growth cones (Igarashi et al., 1996; Dupraz et al., 2009; Bloom and Morgan, 2011; Wang et al., 2011). While the mechanisms by which Rab33a is involved in the axonal transport remain unclear, the GTP dependency of the Rab33a effects observed in this study implies that it acts via a
GTP-dependent interaction with effecter molecules. We reported previously that downregulation of Singar 1 leads to the formation of multiple axons, indicating that Singar 1 is involved in a process underlying the regulation of axon formation (Mori et al., 2007). More recently, we found that Singarl preferentially binds with the GTP-bound form of Rab33a (H. Nakazawa and N. Inagaki, unpublished data), suggesting that it may function as an effector of Rab33a. Detailed analysis of the functional interaction between Rab33a and Singar1 and the identification of additional Rab33a effectors will improve our understanding of the mechanism involved in Rab33a-induced transport of synaptophysinpositive vesicles.

Different Rab proteins reportedly contribute to vesicular trafficking steps underlying neurite outgrowth. For example, Rab27 and Rab11 regulate the axonal transport of vesicles containing the neurotrophin receptor Trk and integrins, molecules that regulate neurite outgrowth (Arimura et al., 2009; Ascaño et al., 2009; Eva et al., 2010). Rab8, Rab11, and Rab5 are also implicated in the regulation of neurite extension (Huber et al., 1995; Shirane and Nakayama, 2006; Liu et al., 2007). We found that Rab33a did not colocalize with vesicles harboring these molecules, suggesting that it is involved in a distinct trafficking route. Given the diversity of vesicular compartments within axons, the details of how membrane pools are transported for axonal extension remain important issues for future investigation.

Rab33a and synaptophysin-positive vesicles accumulate in growing axons, implying their role in the establishment of neuronal polarity. Indeed, neuronal polarization was disrupted when the directional transport of synaptophysin-positive vesicles was impaired by Rab33a RNAi or Rab33a overexpression. The mechanisms by which Rab33a accumulates preferentially in growing axons remain to be analyzed. In neurons, fast anterograde transport is mediated principally by kinesin family proteins (Vallee and Bloom, 1991; Hirokawa and Takemura, 2005). As Rab33a gradually accumulates in axons after the axon is established (stage 4 ), kinesins which accumulate specifically in axons, such as KIF5A, KIF5B, KIF5C, KIF3A/3B, KIF17, KIF13A, and KIF21A (Nakata and Hirokawa, 2003; Jacobson et al., 2006; Huang and Banker, 2011) may be involved in the axonal localization of Rab33a.

In conclusion, the present studies have revealed that Rab33a is a key component of axonal vesicular transport pathways, which contribute to membrane exocytosis at the growth cones. The directional membrane supply from the cell body, together with local membrane endocytosis and recycling (Tojima et al., 2007; Winckler and Yap, 2011), force generation (Suter and Forscher, 2000; Bard et al., 2008; Shimada et al., 2008), and cytoskeletal dynamics (Arimura and Kaibuchi, 2005; Lowery and Van Vactor, 2009; Neukirchen and Bradke, 2011) at the growth cone, may coordinately contribute to axon outgrowth.

\section{References}

Arimura N, Kaibuchi K (2005) Key regulators in neuronal polarity. Neuron 48:881-884.

Arimura N, Kimura T, Nakamuta S, Taya S, Funahashi Y, Hattori A, Shimada A, Ménager C, Kawabata S, Fujii K, Iwamatsu A, Segal RA, Fukuda M, Kaibuchi K (2009) Anterograde transport of TrkB in axons is mediated by direct interaction with Slp1 and Rab27. Dev Cell 16:675-686.

Ascaño M, Richmond A, Borden P, Kuruvilla R (2009) Axonal targeting of Trk receptors via transcytosis regulates sensitivity to neurotrophin responses. J Neurosci 29:11674-11685.

Babbey CM, Ahktar N, Wang E, Chen CCH, Grant BD, Dunn KW (2006) Rab10 regulates membrane transport through early endosomes of polarized Madin-Darby Canine Kidney cells. Mol Biol Cell 17:3156-3175.

Bard L, Boscher C, Lambert M, Mège RM, Choquet D, Thoumine O (2008) 
A molecular clutch between the actin flow and N-cadherin adhesions drives growth cone migration. J Neurosci 28:5879-5890.

Bloom OE, Morgan JR (2011) Membrane trafficking events underlying axon repair, growth, and regeneration. Mol Cell Neurosci 48:339-348.

Bray D (1970) Surface movements during the growth of single explanted neurons. Proc Natl Acad Sci U S A 65:905-910.

Cheng E, Trombetta SE, Kovacs D, Beech RD, Ariyan S, Reyes-Mugica M, McNiff JM, Narayan D, Kluger HM, Picardo M, Halaban R (2006) Rab33A: characterization, expression, and suppression by epigenetic modification. J Invest Dermatol 126:2257-2271.

Craig AM, Banker G (1994) Neuronal polarity. Annu Rev Neurosci $17: 267-310$.

Craig AM, Wyborski RJ, Banker G (1995) Preferential addition of newly synthesized membrane protein at axonal growth cones. Nature 375:592-594.

De Camilli P, Jahn R (1990) Pathways to regulated exocytosis in neurons. Annu Rev Physiol 52:625-645.

Deinhardt K, Salinas S, Verastegui C, Watson R, Worth D, Hanrahan S, Bucci C, Schiavo G (2006) Rab5 and Rab7 control endocytic sorting along the axonal retrograde transport pathway. Neuron 52:293-305.

Dupraz S, Grassi D, Bernis ME, Sosa L, Bisbal M, Gastaldi L, Jausoro I, Cáceres A, Pfenninger KH, Quiroga S (2009) The TC10-Exo70 complex is essential for membrane expansion and axonal specification in developing neurons. J Neurosci 29:13292-13301.

Eva R, Dassie E, Caswell PT, Dick G, ffrench-Constant C, Norman JC, Fawcett JW (2010) Rab11 and its effector Rab coupling protein contribute to the trafficking of beta 1 integrins during axon growth in adult dorsal root ganglion neurons and PC12 cells. J Neurosci 30:11654-11669.

Fletcher TL, Cameron P, De Camilli P, Banker G (1991) The distribution of synapsin I and synaptophysin in hippocampal neurons developing in culture. J Neurosci 11:1617-1626.

Fukuda M (2004) RNA interference-mediated silencing of synaptotagmin IX, but not synaptotagmin I, inhibits dense-core vesicle exocytosis in PC12 cells. Biochem J 380:875-879.

Fukuda M (2008) Regulation of secretory vesicle traffic by Rab small GTPases. Cell Mol Life Sci 65:2801-2813.

Fukuda M, Kobayashi H, Ishibashi K, Ohbayashi N (2011) Genome-wide investigation of the Rab binding activity of RUN domains: development of a novel tool that specifically traps GTP-Rab35. Cell Struct Funct 36:155-170.

Futerman AH, Banker GA (1996) The economics of neurite outgrowththe addition of new membrane to growing axons. Trends Neurosci 19:144-149.

Granseth B, Odermatt B, Royle SJ, Lagnado L (2006) Clathrin-mediated endocytosis is the dominant mechanism of vesicle retrieval at hippocampal synapses. Neuron 51:773-786.

Hattula K, Furuhjelm J, Tikkanen J, Tanhuanpää K, Laakkonen P, Peränen J (2006) Characterization of the Rab8-specific membrane traffic route linked to protrusion formation. J Cell Sci 119:4866-4877.

Hémar A, Olivo JC, Williamson E, Saffrich R, Dotti CG (1997) Dendroaxonal transcytosis of transferrin in cultured hippocampal and sympathetic neurons. J Neurosci 17:9026-9034.

Hirokawa N, Takemura R (2005) Molecular motors and mechanisms of directional transport in neurons. Nat Rev Neurosci 6:201-214.

Huang CF, Banker G (2012) The translocation selectivity of the kinesins that mediate neuronal organelle transport. Traffic 13:549-564.

Huber LA, Dupree P, Dotti CG (1995) A deficiency of the small GTPase rab8 inhibits membrane traffic in developing neurons. Mol Cell Biol 15:918-924.

Igarashi M, Kozaki S, Terakawa S, Kawano S, Ide C, Komiya Y (1996) Growth cone collapse and inhibition of neurite growth by Botulinum neurotoxin C1: a t-SNARE is involved in axonal growth. J Cell Biol 134:205-215.

Igarashi M, Tagaya M, Komiya Y (1997) The soluble N-ethylmaleimidesensitive factor attached protein receptor complex in growth cones: molecular aspects of the axon terminal development. J Neurosci 17:1460-1470.

Inagaki N, Chihara K, Arimura N, Ménager C, Kawano Y, Matsuo N, Nishimura T, Amano M, Kaibuchi K (2001) CRMP-2 induces axons in cultured hippocampal neurons. Nat Neurosci 4:781-782.

Itoh T, Satoh M, Kanno E, Fukuda M (2006) Screening for target Rabs of
TBC (Tre-2/Bub2/Cdc16) domain-containing proteins based on their Rab-binding activity. Genes Cells 11:1023-1037.

Jacobson C, Schnapp B, Banker GA (2006) A change in the selective translocation of the Kinesin-1 motor domain marks the initial specification of the axon. Neuron 49:797-804.

Jahn R, Schiebler W, Ouimet C, Greengard P (1985) A 38,000-dalton membrane protein (p38) present in synaptic vesicles. Proc Natl Acad Sci U S A 82:4137-4141.

Kaether C, Skehel P, Dotti CG (2000) Axonal membrane proteins are transported in distinct carriers: a two-color video microscopy study in cultured hippocampal neurons. Mol Biol Cell 11:1213-1224.

Kelly RB (1991) Secretory granule and synaptic vesicle formation. Curr Opin Cell Biol 3:654-660.

Kobayashi T, Hori Y, Ueda N, Kajiho H, Muraoka S, Shima F, Kataoka T, Kontani K, Katada T (2009) Biochemical characterization of missense mutations in the Arf/Arl-family small GTPase Arl6 causing Bardet-Biedl syndrome. Biochem Biophys Res Commun 381:439-442.

Kunda P, Paglini G, Quiroga S, Kosik K, Caceres A (2001) Evidence for the involvement of Tiam1 in axon formation. J Neurosci 21:2361-2372.

Lee MS, Jun DH, Hwang CI, Park SS, Kang JJ, Park HS, Kim J, Kim JH, Seo JS, Park WY (2006) Selection of neural differentiation-specific genes by comparing profiles of random differentiation. Stem Cells 24:1946-1955.

Leterrier C, Lainé J, Darmon M, Boudin H, Rossier J, Lenkei Z (2006) Constitutive activation drives compartment-selective endocytosis and axonal targeting of type 1 cannabinoid receptors. J Neurosci 26:3141-3153.

Linstedt AD, Hauri HP (1993) Giantin, a novel conserved Golgi membrane protein containing a cytoplasmic domain of at least $350 \mathrm{kDa}$. Mol Biol Cell 4:679-693.

Liu J, Lamb D, Chou MM, Liu YJ, Li G (2007) Nerve growth factormediated neurite outgrowth via regulation of Rab5. Mol Biol Cell 18:1375-1384.

Lockerbie RO, Miller VE, Pfenninger KH (1991) Regulated plasmalemmal expansion in nerve growth cones. J Cell Biol 112:1215-1227.

Lombardi D, Soldati T, Riederer MA, Goda Y, Zerial M, Pfeffer SR (1993) Rab9 functions in transport between late endosomes and the trans Golgi network. EMBO J 12:677-682.

Lowery LA, Van Vactor D (2009) The trip of the tip: understanding the growth cone machinery. Nat Rev Mol Cell Biol 10:332-343.

Luzio JP, Brake B, Banting G, Howell KE, Braghetta P, Stanley KK (1990) Identification, sequencing and expression of an integral membrane protein of the trans-Golgi network (TGN38). Biochem J 270:97-102.

McDonald NA, Henstridge CM, Connolly CN, Irving AJ (2007) An essential role for constitutive endocytosis, but not activity, in the axonal targeting of the CB1 cannabinoid receptor. Mol Pharmacol 71:976-984.

Miesenböck G, De Angelis DA, Rothman JE (1998) Visualizing secretion and synaptic transmission with $\mathrm{pH}$-sensitive green fluorescent proteins. Nature 394:192-195.

Mori T, Wada T, Suzuki T, Kubota Y, Inagaki N (2007) Singar1, a novel RUN domain-containing protein, suppresses formation of surplus axons for neuronal polarity. J Biol Chem 282:19884-19893.

Nakamura N, Rabouille C, Watson R, Nilsson T, Hui N, Slusarewicz P, Kreis TE, Warren G (1995) Characterization of a cis-Golgi matrix protein, GM130. J Cell Biol 131:1715-1726.

Nakata T, Hirokawa N (2003) Microtubules provide directional cues for polarized axonal transport through interaction with kinesin motor head. J Cell Biol 162:1045-1055.

Nakata T, Terada S, Hirokawa N (1998) Visualization of the dynamics of synaptic vesicle and plasma membrane proteins in living axons. J Cell Biol 140:659-674

Neukirchen D, Bradke F (2011) Neuronal polarization and the cytoskeleton. Semin Cell Dev Biol 22:825-833.

Niwa H, Yamamura K, Miyazaki J (1991) Efficient selection for highexpression transfectants with a novel eukaryotic vector. Gene 108:193-199.

Pfeffer SR (2001) Rab GTPases: specifying and deciphering organelle identity and function. Trends Cell Biol 11:487-491.

Pfenninger KH (2009) Plasma membrane expansion: a neuron's Herculean task. Nat Rev Neurosci 10:251-261.

Pfenninger KH, Friedman LB (1993) Sites of plasmalemmal expansion in growth cones. Brain Res Dev Brain Res 71:181-192.

Phelan P, Gordon-Weeks PR (1992) Widespread distribution of synapto- 
physin, a synaptic vesicle glycoprotein, in growing neurites and growth cones. Eur J Neurosci 4:1180-1190.

Régnier-Vigouroux A, Tooze SA, Huttner WB (1991) Newly synthesized synaptophysin is transported to synaptic-like microvesicles via constitutive secretory vesicles and the plasma membrane. EMBO J 10:3589-3601.

Robinson MS (1990) Cloning and expression of gamma-adaptin, a component of clathrin-coated vesicles associated with the Golgi apparatus. J Cell Biol 111:2319-2326.

Rogalski AA, Singer SJ (1984) Associations of elements of the Golgi apparatus with microtubules. J Cell Biol 99:1092-1100.

Roland JT, Kenworthy AK, Peranen J, Caplan S, Goldenring JR (2007) Myosin $\mathrm{Vb}$ interacts with Rab8a on a tubular network containing EHD1 and EHD3. Mol Biol Cell 18:2828-2837.

Rosso S, Bollati F, Bisbal M, Peretti D, Sumi T, Nakamura T, Quiroga S, Ferreira A, Cáceres A (2004) LIMK1 regulates Golgi dynamics, traffic of Golgi-derived vesicles, and process extension in primary cultured neurons. Mol Biol Cell 15:3433-3449.

Saito S, Fujita T, Komiya Y, Igarashi M (1992) Biochemical characterization of nerve growth cones isolated from both fetal and neonatal rat forebrains: the growth cone particle fraction mainly consists of axonal growth cones in both stages. Brain Res Dev Brain Res 65:179-184.

Sampo B, Kaech S, Kunz S, Banker G (2003) Two distinct mechanisms target membrane proteins to the axonal surface. Neuron 37:611-624.

Sann S, Wang Z, Brown H, Jin Y (2009) Roles of endosomal trafficking in neurite outgrowth and guidance. Trends Cell Biol 19:317-324.

Satoh T, Kaziro Y (1995) Measurement of Ras-bound guanine nucleotide in stimulated hematopoietic cells. Methods Enzymol 255:149-155.

Shimada T, Toriyama M, Uemura K, Kamiguchi H, Sugiura T, Watanabe N, Inagaki N (2008) Shootin 1 interacts with actin retrograde flow and L1CAM to promote axon outgrowth. J Cell Biol 181:817-829.

Shirane M, Nakayama KI (2006) Protrudin induces neurite formation by directional membrane trafficking. Science 314:818-821.

Sönnichsen B, De Renzis S, Nielsen E, Rietdorf J, Zerial M (2000) Distinct membrane domains on endosomes in the recycling pathway visualized by multicolor imaging of Rab4, Rab5, and Rab11. J Cell Biol 149:901-914.

Suter DM, Forscher P (2000) Substrate-cytoskeletal coupling as a mecha- nism for the regulation of growth cone motility and guidance. J Neurobiol 44:97-113.

Tojima T, Akiyama H, Itofusa R, Li Y, Katayama H, Miyawaki A, Kamiguchi H (2007) Attractive axon guidance involves asymmetric membrane transport and exocytosis in the growth cone. Nat Neurosci 10:58-66.

Toriyama M, Shimada T, Kim KB, Mitsuba M, Nomura E, Katsuta K, Sakumura Y, Roepstorff P, Inagaki N (2006) Shootin1: a protein involved in the organization of an asymmetric signal for neuronal polarization. J Cell Biol 175:147-157.

Tsuboi T, Fukuda M (2006) Rab3A and Rab27A cooperatively regulate the docking step of dense-core vesicle exocytosis in PC12 cells. J Cell Sci 119:2196-2203.

Ullrich O, Reinsch S, Urbé S, Zerial M, Parton RG (1996) Rab11 regulates recycling through the pericentriolar recycling endosome. J Cell Biol 135:913-924.

Vallee RB, Bloom GS (1991) Mechanisms of fast and slow axonal-transport. Annu Rev Neurosci 14:59-92.

van der Sluijs P, Hull M, Webster P, Mâle P, Goud B, Mellman I (1992) The small GTP-binding protein rab4 controls an early sorting event on the endocytic pathway. Cell 70:729-740.

Wang T, Liu Y, Xu XH, Deng CY, Wu KY, Zhu J, Fu XQ, He M, Luo ZG (2011) Lgl1 activation of Rab10 promotes axonal membrane trafficking underlying neuronal polarization. Dev Cell 21:431-444.

Wiedenmann B, Franke WW (1985) Identification and localization of synaptophysin, an integral membrane glycoprotein of Mr 38,000 characteristic of presynaptic vesicles. Cell 41:1017-1028.

Winckler B, Yap CC (2011) Endocytosis and endosomes at the crossroads of regulating trafficking of axon outgrowth-modifying receptors. Traffic 12:1099-1108.

Wisco D, Anderson ED, Chang MC, Norden C, Boiko T, Fölsch H, Winckler B (2003) Uncovering multiple axonal targeting pathways in hippocampal neurons. J Cell Biol 162:1317-1328.

Zerial M, McBride H (2001) Rab proteins as membrane organizers. Nat Rev Mol Cell Biol 2:107-117.

Zheng JY, Koda T, Arimura Y, Kishi M, Kakinuma M (1997) Structure and expression of the mouse S10 gene. Biochim Biophys Acta 1351:47-50. 